\title{
Deep Dynamic Stall and Active Aerodynamic Modification on a S833 Airfoil using Pitching Trailing Edge Flap
}

Wind Engineering

XX(X):1-20

(C)The Author(s) 2016

Reprints and permission:

sagepub.co.uk/journalsPermissions.nav

DOI: $10.1177 /$ ToBeAssigned

www.sagepub.com/

SAGE

\author{
Farid Samara ${ }^{1}$ and David A. Johnson ${ }^{2}$
}

\begin{abstract}
Due to the dynamic nature of the wind resource, wind turbine blades are subjected to significant variation in flow parameters such as angle of attack $(\alpha)$. In some cases, the occurrence of dynamic stall on wind turbine blades causes load fluctuation which leads to material fatigue that tend to decrease the lifespan of the blades. In this study, the influence of a trailing edge flap (TEF) on dynamic stall effects is investigated at high $\alpha$ typical of wind turbines but atypical elsewhere. Pitching of the TEF was found to have a significant impact on the dynamic stall hysteresis loops responsible for the load fluctuation. Frequency analysis showed that the TEF was capable of reducing the cyclic fluctuation in the coefficient of lift and root bending moment by at least $26 \%$ and $24 \%$ respectively. These results are a significant contribution towards understanding the advantages of using TEFs and how implementing them will reduce wind turbine blade load fluctuations.
\end{abstract}

\section{Keywords}

Trailing Edge Flap (TEF), Dynamic Stall, Experimental Wind Tunnel, Leading Edge Vortex, Wind Turbine Load Alleviation

\section{Introduction}

Wind energy, an established renewable source of energy, has evolved over the last several decades to produce a significant share of electricity generation across the world. The success of horizontal axis wind turbines (HAWT) is due to innovation that has decreased the electricity production cost per kWh (Dykes et al., 2019). It is understood that most wind turbines spend most of the time in a relatively unsteady flow environment caused by many factors including wind shear, rotor misalignment, and turbulence of the wind resource to name a few (Shipley et al., 1995; Leishman, 2006). This flow unsteadiness causes cyclic loading on the blades that leads to material fatigue which decreases the lifespan of the blades. Fatigue life governs the design factors for larger wind turbines, so it is important to minimize the cyclic loading (Barlas and van Kuik, 2010). For this reason, it is imperative to understand and reduce cyclic loading on the blades to increase turbine lifespan and decrease the cost of generated energy. To reduce cyclic loading and material fatigue, active aerodynamic modification of the blade, also known as Smart Rotor, could be used. Different active aerodynamic control strategies have been summarized and reviewed by Barlas and van Kuik (2010). They found that trailing edge flaps (TEFs) were the most efficient of the control strategies tested because of their control authority over the coefficients of lift and drag, linearity, high frequency response and simplicity of use. A TEF is achieved by hinging the trailing edge of the airfoil at a specific location.

Cyclic loading on a rotating turbine blade tends to be caused by a variation in the blade local angle of attack, $\alpha$, and that variation is due to the unsteady flow environment mentioned. In some

\footnotetext{
${ }^{1}$ Ph.D., Wind Energy Group, Mechanical and Mechatronics Engineering, University of Waterloo, Waterloo, Canada; fsamara@uwaterloo.ca ${ }^{2}$ Professor, Wind Energy Group, Mechanical and Mechatronics Engineering, University of Waterloo, Waterloo, Canada; david.johnson@uwaterloo.ca

Corresponding author:

Farid Samara, Wind Energy Group, Mechanical and Mechatronics Engineering, University of Waterloo, 200 University Ave W, Waterloo, Canada N2L 3G1

Email: fsamara@uwaterloo.ca
} 


\begin{tabular}{|llll|}
\hline \multicolumn{2}{|l|}{ Nomenclature } & & \\
$\alpha$ & angle of attack, deg & & \\
$\alpha_{a m p}$ & pitching semi-amplitude, deg & $C_{M}$ & coefficient of moment, [-] \\
$\alpha_{F}$ & flap angle of attack, deg & $C_{p}$ & coefficient of pressure, [-] \\
$\alpha_{\text {mean }, F}$ flap pitching mean geometric angle of attack,, & $f_{F}$ & frequency of oscillation, $\mathrm{s}^{-1}$ \\
& deg & $f_{p}$ & pitch frequency of oscillation, $\mathrm{s}^{-1}$ \\
$\alpha_{\text {mean }}$ & pitching mean geometric angle of attack, deg & $k$ & reduced frequency $\left(k=\pi c f / U_{\infty}\right)$ \\
$\gamma$ & yaw angle, deg & $U_{\infty}$ & free stream velocity, m.s $\mathrm{s}^{-1}$ \\
$\phi$ & phase shift, rad & $\mathrm{c}$ & chord, m \\
$\rho_{\infty}$ & free stream air density, kg.m ${ }^{-3}$ & $\mathrm{cw}$ & clockwise \\
$\tau$ & period of the cycle, $\mathrm{s}$ & $\mathrm{cww}$ & counter-clockwise \\
$C_{D}$ & coefficient of drag, [-] & PSD & Power Spectral Density \\
$C_{L}$ & coefficient of Lift, [-] & $\mathrm{STD}$ & standard deviation \\
& & & \\
\hline
\end{tabular}

cases, for example when the turbine is yawed, the $\alpha$ variation tends to be cyclic in nature with a frequency of once per rotor revolution (Burton et al., 2011). This cyclic variation in $\alpha$ not only causes load variation based on steady flow dynamics but also on unsteady flow dynamics or dynamic stall under specific conditions. A $2 \mathrm{D}$ airfoil undergoing pitching in a wind tunnel could be used to study and simulate dynamic stall. In this experimental campaign, the use of a TEF on a pitching airfoil is investigated to reduce cyclic loading that could be caused by the occurrence of dynamic stall on the blade. It is then important to understand the characteristics and formation of dynamic stall in this application.

When the airfoil pitching range exceeds the static stall angle by a few degrees, then dynamic stall ensues according to McCroskey (1982). Dynamic stall is characterized by extreme hysteresis and non-linear cycles in the coefficient of lift $\left(C_{L}\right)$, coefficient of moment $\left(C_{M}\right)$ and coefficient of drag $\left(C_{D}\right)$ (McCroskey, 1982). The dynamic stall cycle could be divided into four sections as seen in Figure 1: a) start of pitch-up motion, b) flow separation, c) start of pitch-down motion, and d) flow reattachment. During pitch-up motion, as $C_{L}$ increases steadily past the static stall angle, there is a sudden spike in $C_{L}$ caused by the formation of a leading edge vortex (LEV) also known as the dynamic stall vortex (Corke and Thomas, 2015) shown in Figure 1. The LEV is generated at the leading edge (LE) and convects downstream along the airfoil towards the trailing edge (TE). The LEV generation and convection produces values of $C_{L}, C_{M}$ and $C_{D}$ that are much higher than their static counterparts (McCroskey, 1982). After the LEV flows past the TE the flow separates leading to a severe loss in lift, an increase in nose-down pitching moment and an increase in drag. In some cases a secondary LEV is formed and shed, leading to another $C_{L}$ peak (Leishman, 1990). Gharali and Johnson $(2013,2015)$ and Gharali et al. (2015) reported the formation of a secondary LEV and in some cases smaller vortices after that. The secondary LEV tends to be weaker in magnitude when compared to the primary LEV. Lee and Gerontakos (2004) have also reported the formation of a secondary LEV. Typically, during pitch-down motion, the airfoil is stalled even when $\alpha$ is below the static stall angle. The flow then reattaches to the surface as $\alpha$ decreases and the cycle starts again (Baik et al., 2012; Cebeci et al., 2005; Pitt Ford and Babinsky, 2013). Leishman (1990) and recently Masdari et al. (2018) studied the effects of mean $\alpha$, pitching amplitude, and reduced frequency on dynamic stall. Surface pressure measurements have been used in the past to determine the formation and development of dynamic stall and the LEV by Leishman (1990), McCroskey et al. (1976) and others. These studies show how the LEV could be identified using surface pressure measurements alone.

While the airfoil is pitching past the static stall angle and dynamic stall is present, aeroelastic stability problems could arise. Aeroelastic divergence or stall flutter is promoted when torsional damping (defined by the line integral $\oint C_{m}(\alpha) d \alpha$ ) is negative (Leishman, 2006). A counter-clockwise (ccw) loop in the $C_{M}-\alpha$ plot indicates that the torsional damping is positive while a clockwise (cw) loop indicates that the torsional damping is negative. Negative 


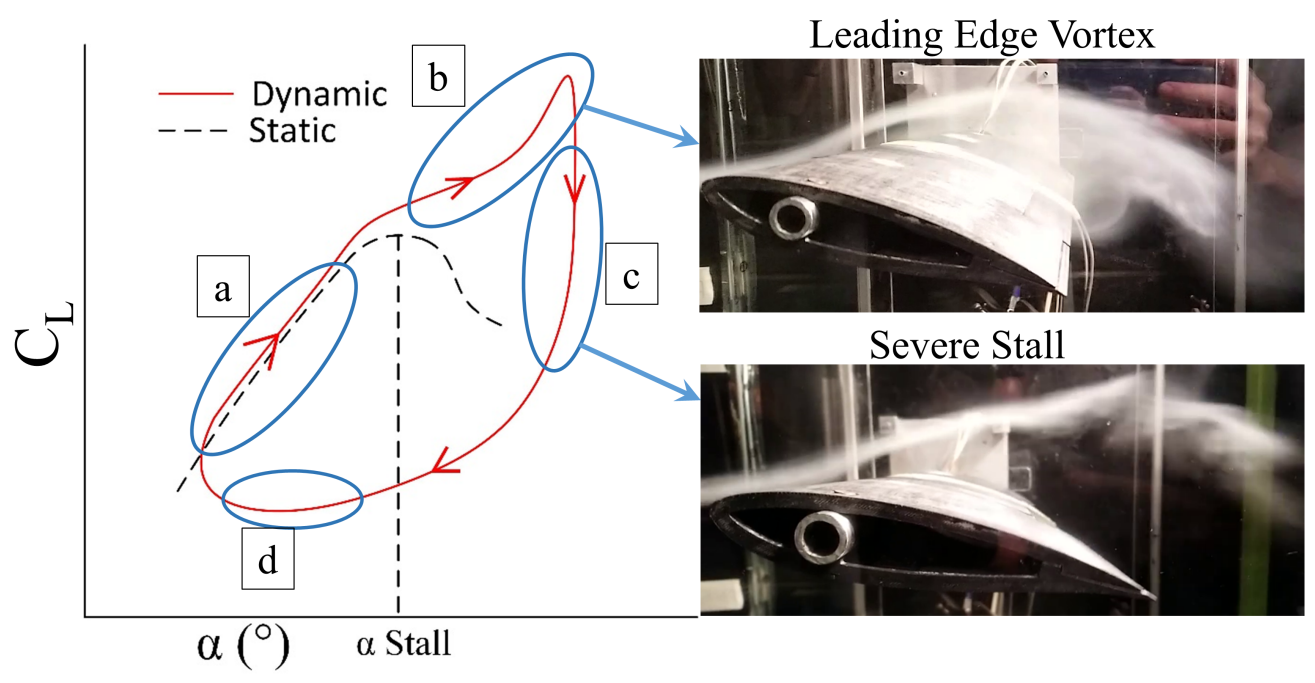

Figure 1. A schematic illustrating the influence of the dynamic stall cycle on the airfoil $C_{L}$. The cycle is divided into four sections: a) start of pitch-up motion, b) flow separation, c) start of pitch-down motion and d) flow re-attachment. The pictures, taken during the experimental campaign, help visualize the LEV re-circulation and stall.

damping may lead to structural vibrations and rapid mechanical failure if the structural dynamics permit (Corke and Thomas, 2015; Green et al., 2011). Using a TEF to mitigate negative damping without significant loss of lift would benefit the energy capture and turbine system greatly and would ensure limited flutter on the airfoil (Green et al., 2011).

TEF also known as plain flap, or aileron has been used in the past in the aviation industry to modify the aerodynamic characteristics and obtain higher $C_{L}$ values (Abbott and Doenhoff, 1959). A plain flap is described to be a relatively crude device and is likely to lead to some form of boundary layer separation (Houghton and Carpenter, 2002). This means that there is always a possibility of partial separation when the flap is deflected. Abbott and Greenberg (1939) studied a plain flap of $20 \%$ chord on a static NACA 23012 airfoil with a large range of flap and pitch angles. They found that the TEF was capable of shifting the entire $C_{L}-\alpha$ data up and down in both pre-stall and stall flow equally. The stall angle was also shifted slightly to a lower or higher angle depending on the flap position. The flap not only has influence over $C_{L}$, but also has a large impact on $C_{M}$ and $C_{D}$. Their results showed that a positive flap angle, defined in this paper as deflection towards the suction side (See Figure 4), increased $C_{D}$ significantly but a negative flap angle only had a slight impact on $C_{D}$. Jacobs and Pinkerton (1931) showed that the TEF changes the static surface pressure distribution not only at the TE but at the LE as well. Most of the prior research regarding flaps is focused on negative flap angles to achieve higher $C_{L}$ values of relevance to the aviation industry. When applying flaps on wind turbines it is as important to equally understand the aerodynamics of the airfoil in positive and negative flap angles alike to reduce the cyclic loading on the airfoil. The Static Airfoil Characterization section of this paper focuses on understanding how large flap angles (higher than $\pm 20^{\circ}$ ) influence the aerodynamics of a wind turbine airfoil in static conditions.

In recent years, TEFs have been investigated and used to manipulate or reduce the impact of dynamic stall on airfoils. In most cases, the TEF would be oscillating at a frequency equal to that of the airfoil pitching frequency but with a phase shift defined as $\phi$ that influences the shape of the dynamic stall loops. Having the appropriate $\phi$ is key to reducing the load fluctuation due to dynamic stall. Choosing the appropriate $\phi$ is dependent on the oscillating dynamics such as the mean $\alpha$, pitching amplitude, and reduced frequency. Lee and Gerontakos (2006); Lee and Su (2011) investigated how a TEF can influence the formation and detachment of LEV. They concluded that the TEF was not capable of controlling the formation and detachment of the LEV but was capable of controlling the LEV magnitude. Green et al. (2011) discuss the benefit of TEF and how it can influence dynamic stall behavior. They reported that flap deflection was capable of reducing the hysteresis cycle in the lift and moment data. Furthermore, with the appropriate $\phi$, negative torsional damping could be reduced or completely eliminated. Raiola et al. (2018) showed that a TEF on a NACA0015 airfoil was capable of controlling the loads generated by dynamic stall and the LEV. Troldborg (2005) was able to use the TEF motion to counteract the oscillating pitch motion and obtain smooth lift data on 
the airfoil. Zanotti and Gibertini (2018) investigated the use of an active Gurney flap for dynamic stall control on a pitching airfoil and found that the flap was capable of reducing the amplitude of dynamic stall. Feszty et al. (2004) and Shehata et al. (2018) showed that pulsing the TEF was capable of improving wind turbine rotor performance and removing negative effects of dynamic stall. From the literature review presented, it was found that more data is needed regarding dynamic stall behavior on wind turbine specific airfoils and cambered airfoils with a TEF.

In some operating conditions, a percentage of the wind turbine blade span could be experiencing $\alpha$ values higher than $30^{\circ}$. Gallant and Johnson (2017) measured $\alpha$ on a wind turbine blade operating under yaw conditions to be oscillating around a mean $\alpha$ of $25^{\circ}$ with an amplitude of $5^{\circ}$. Most of the papers and references presented so far study the dynamic stall cycle where the maximum pitch angle is $25^{\circ}$. In this current paper, deep dynamic stall is investigated on a cambered airfoil with a TEF where the maximum pitch angle reaches $30^{\circ}$ while the airfoil is oscillating. Few studies investigate deep dynamic stall on an airfoil and present data for pitch angles past $25^{\circ}$ (Leishman, 1990; McCroskey, 1981; Gerontakos, 2004; Zanotti, 2012; Zanotti and Gibertini, 2013). The literature was found to lack data on a cambered airfoil equipped with a TEF and operating in deep stall. This paper builds on prior studies and specifically discusses the S833 cambered airfoil that was designed for small scale wind turbines (Somers, 2005). An experimental campaign was initiated to study a TEF on a wind turbine beginning with characterizing a 2D airfoil model with a TEF in a closed loop wind tunnel at a chord $R e=170 \times 10^{3}$ and a reduced frequency $(k)$ of 0.06 and 0.1. Time resolved surface pressure measurements on the airfoil element have been obtained for different dynamic cases and flap positions. A few of the novel contributions introduced in this paper are: the design and actuation of a TEF on a small airfoil section, and dynamic stall studies on a wind turbine specific airfoil in deep dynamic stall cases with an oscillating TEF.

\section{Experimental Setup}

First, the instrumented airfoil model is explained briefly along with the wind tunnel setup. Secondly, the airfoil motion and different test cases are presented. Finally, the validation for the differential pressure measurement technique is discussed.

\section{Wind Tunnel Setup}

The experimental campaign was carried out in the closed loop wind tunnel at the University of Waterloo. The wind tunnel has a contraction ratio of 9:1 and a cross section of $0.61 \mathrm{~m}$ square. The uniformity was found to be within $\pm 0.4 \%$ in the spanwise and vertical direction. The turbulence intensity was $0.1 \%$. The free-stream velocity was set by the static pressure drop across the contraction with an uncertainty of less than $2.5 \%$. The blockage ratio was around $8 \%$. The wind tunnel velocity or the free stream velocity, $U_{\infty}$, was set to $15 \mathrm{~m} / \mathrm{s}$ resulting in a chord-based Reynolds number $(\mathrm{Re})$ of $170 \times 10^{3}$. This velocity was based upon a compromise between high Reynolds number and an achievable pitch frequency to achieve dynamic stall behavior on the airfoil.

The NREL S833 airfoil (Somers, 2005), designed for small wind turbines, was used for the experiments. The flap width was chosen to be $20 \%$ of the $178 \mathrm{~mm}$ model chord. The flap span was not capable of occupying the entire model span due to geometric restrictions including bearings and flap motor. Thus, the flap span occupied $60 \%$ of the $584 \mathrm{~mm}$ model span. To incorporate the sensors and actuators the aerodynamic airfoil segment was 3D printed. A stainless steel spar at the quarter chord was used to support the 3D printed airfoil segments and hold all the components together. The airfoil was then cantilevered and supported on the motor drive side in the wind tunnel. Two strain gage groups were used to measure the strain at the airfoil support to study the load on the entire airfoil section. Precision strain gages (Omega SGD-7/100 0-DY11) were calibrated and connected in a full-bridge configuration to increase sensitivity and reduce temperature dependence. A schematic of the experimental setup is shown in Figure 2. The designed model was capable of housing the TEF actuator and controller inside the airfoil. The airfoil internal arrangement of the flap motion control could be seen in Figure 3.

To determine the coefficient of lift and moment, the phase-averaged pressure was integrated from the 54 surface pressure taps located on an aluminum airfoil section at midspan with 4 of those pressure taps located on the flap. The aluminum pressure tap section was placed at the center of the wind tunnel section sandwiched between the 3D printed airfoil pieces. To measure the surface pressure, 27 differential pressure transducers (All Sensors model $120 \mathrm{cmH} 2 \mathrm{O}-$ D1-4V-MINI) were used to measure the differential pressure between the suction and pressure side of the airfoil at the same chordwise location simultaneously. This technique was successfully used by Raiola et al. (2018) on a symmetric airfoil. To validate this technique so it could be used on cambered airfoils, Samara and Johnson (2020a) conducted a set of experiments to compare differential pressure measurements 


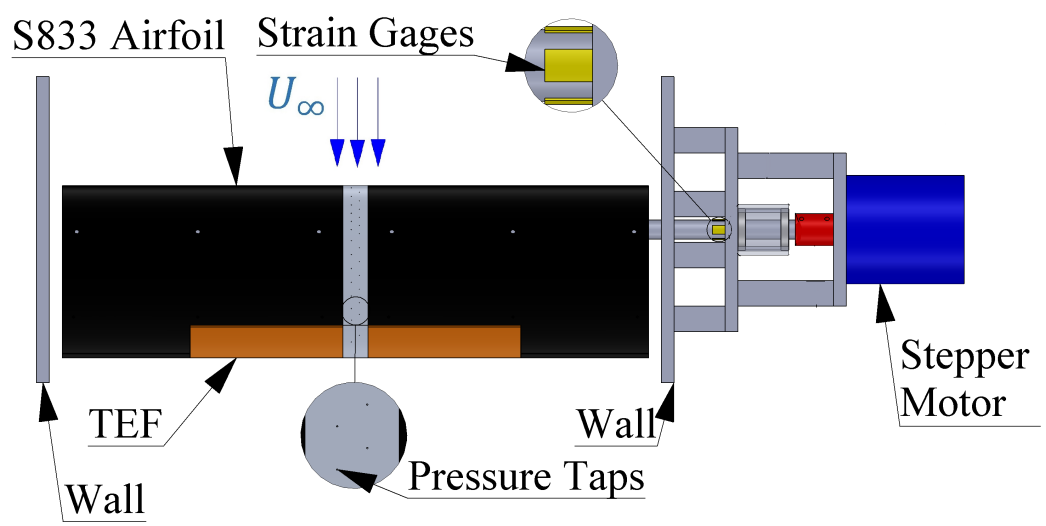

Figure 2. A plan view schematic showing how the airfoil was setup inside the wind tunnel for characterization.

to single ended pressure measurements. They concluded that both techniques are valid to study the patterns in $C_{L}$ and $C_{M}$ on cambered airfoils and the maximum error between both techniques is less than $4 \%$. The 3D printed airfoil model was capable of housing the pressure transducers and all the tubing needed to connect the transducers to the pressure taps. Refer to Figure 3 for more clarification. The coefficient of pressure $\left(\Delta C_{p}\right)$ is calculated as $\Delta C_{p}=\left(p_{\text {suction side }}-p_{\text {pressure side }}\right) /\left(\frac{1}{2} \rho_{\infty} U_{\infty}^{2}\right)$ where $\rho_{\infty}$ is the free stream fluid density. The average representative uncertainty in $C_{L}$ or $C_{M}$ was calculated to be \pm 0.069 and \pm 0.014 respectively. Uncertainty in $\Delta C_{p}$ was calculated to be around 0.085 . The uncertainty was based on the error in the pressure transducers and wind tunnel velocity. The uncertainty in $\alpha$ and TEF angle is $\pm 0.1^{\circ}$ and $\pm 2^{\circ}$ respectively. More details regarding the experimental setup could be found in Samara and Johnson ((Samara and Johnson, 2018, 2020b; Samara, 2020)).

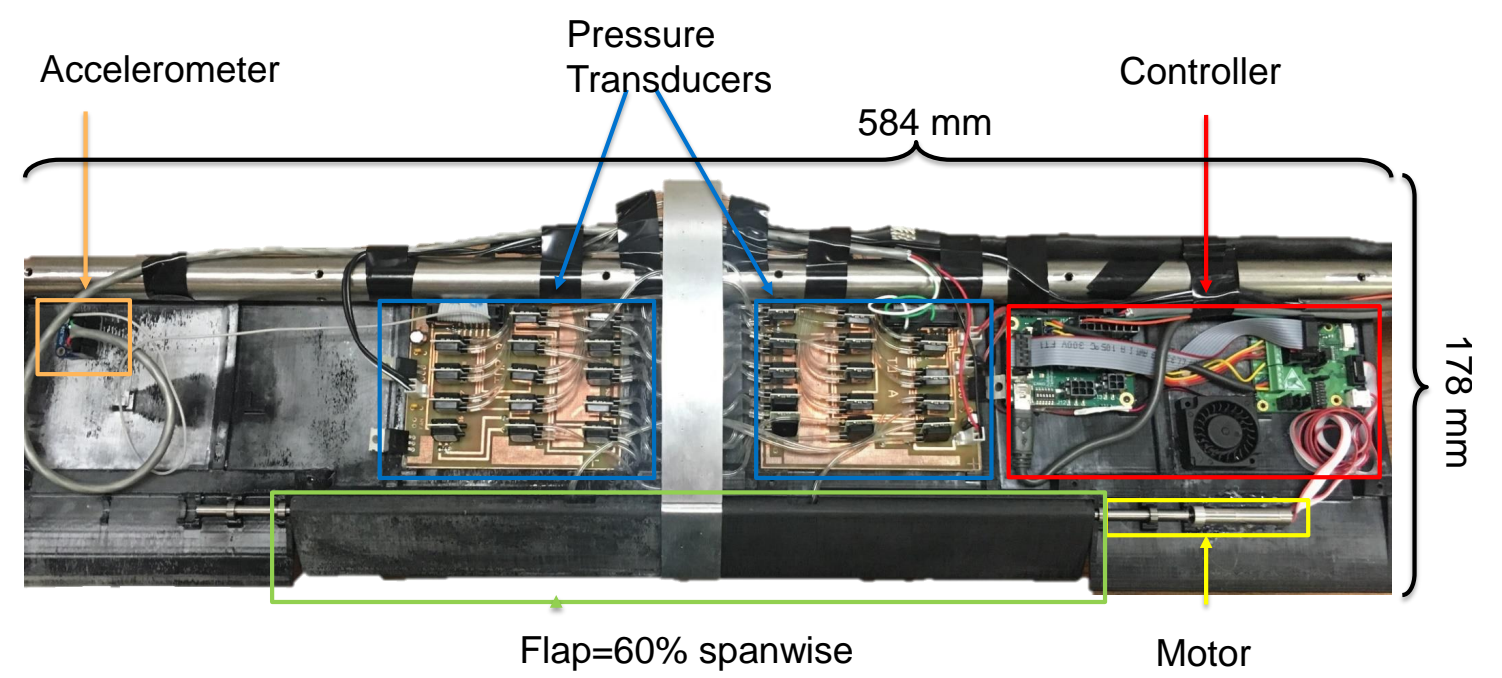

Figure 3. Internal organization of the airfoil showing the sensors used and the TEF actuation system with the airfoil cover removed.

\section{Airfoil Motion}

On a rotating wind turbine blade, one mechanism by which the $\alpha$ varies as the blade rotates occurs when the turbine is yawed. Morote (2016) and Burton et al. (2011) modeled the $\alpha$ variation as a function of azimuth position. Gallant and Johnson (2017) compared those previous two models to experimental data on a $3.4 \mathrm{~m}$ diameter wind turbine. From the models and experiments it was found that the $\alpha$ variation is very similar to a sine function when the turbine 
is yawed. To simulate the dynamic forces on a yawed wind turbine blade, $\alpha(t)$ follows a sine function as reported by Gharali and Johnson (2015) and described in the following equation:

$$
\alpha(t)=\alpha_{\text {mean }}+\alpha_{a m p} \sin \left(2 \pi f_{p} t\right)
$$

where $\alpha_{\text {mean }}$ is the mean geometric angle of attack of the airfoil, $\alpha_{a m p}$ is the semi-amplitude for the pitch motion and $f_{p}$ is the airfoil oscillation frequency. To study the influence that the TEF has on the aerodynamic forces on the airfoil, the flap angle oscillation frequency, $f_{F}$, is the same as the pitch frequency $f_{p}$. The following equation describes the motion for the flap angle, $\alpha_{F}(\mathbf{t})$ :

$$
\alpha_{F}(t)=\alpha_{a m p, F} \sin \left(2 \pi f_{F} t+\phi\right)
$$

where $\alpha_{a m p, F}$ is the TEF semi-amplitude and $\phi$ is the phase shift with respect to the pitch motion. Figure 4 shows the positive direction for the pitch and flap angles. There are three airfoil parameters that influence the dynamic stall or unsteady loading: airfoil shape, mean pitching angle/amplitude, and the reduced frequency $k=\pi c f / U_{\infty}$ ( $c$ is the chord, $f$ is the frequency where it is equal to $f_{p}$ in this experimental setup and $U_{\infty}$ is the freestream velocity) (Carr et al., 1977; McCroskey, 1982).

Corke and Thomas (2015) defined the flow field to be unsteady when $k$ is higher than 0.06 otherwise unsteady effects can be neglected. The system in this study is operated at two different $k$ values of 0.06 and 0.1 to represent the $\alpha$ variation at two different spanwise $r / R$ (where $r$ is the radius of a point on the blade and $R$ is the blade tip radius) locations on a yawed wind turbine blade. The blade element section studied here was subsequently installed on a $3.6 \mathrm{~m}$ diameter wind turbine rotating at a speed of $200 \mathrm{rpm}$ and operating at a tip speed ratio of 3.5. On the turbine, $k$ decreases when the blade element is moved away from the hub. A $k$ value of 0.06 would occur closer to the blade tip $(\mathrm{r} / \mathrm{R}=0.82)$ while $k=0.1$ would occur closer to the blade center $(\mathrm{r} / \mathrm{R}=0.66)$. A $k$ value of 0.06 and 0.1 would represent a local tip speed ratio of 2.87 and 2.31 respectively. More information about the wind turbine setup could be found in Samara and Johnson (2020b). The different test cases reported here are shown in Table 1 . Test case $1 a$ and $1 b$ represent a reduced frequency of 0.06 and 0.1 respectively while $\alpha_{a m p}=10^{\circ}$ and $\alpha_{\text {mean }}=20^{\circ}$. On the other hand, test case 2, represents $k=0.1, \alpha_{a m p}=5^{\circ}$ and $\alpha_{\text {mean }}=25^{\circ}$. Conditions for test 1 causes the airfoil to go into deep dynamic stall while allowing the flow over the airfoil to reattach during the cycle. In test 2 , the airfoil is oscillating past the static stall angle thus the flow does not reattach during the cycle.

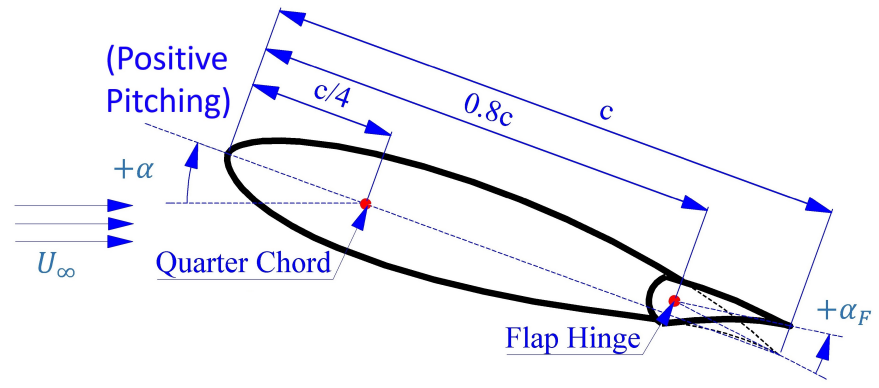

Figure 4. A sketch illustrating the airfoil and flap degrees of freedom. $+\alpha$ positive angle of attack; $+\alpha_{F}$ positive flap angle; $U_{\infty}$ freestream velocity

Table 1. Wind tunnel dynamic airfoil oscillation test cases

\begin{tabular}{lccccccc}
\hline \hline Test No & $f_{p}(\mathrm{~Hz})$ & $k$ & $f_{F}(\mathrm{~Hz})$ & $\alpha_{\text {mean }}\left({ }^{\circ}\right)$ & $\alpha_{a m p}\left(^{\circ}\right)$ & $\alpha_{a m p, F}\left(^{\circ}\right)$ & $\phi$ \\
\hline \hline Case 1a & 1.61 & 0.06 & {$[0,1.61]$} & 20 & 10 & 20 & {$[0: 0.5: 1.5] \pi$} \\
\hline Case 1b & 2.68 & 0.1 & {$[0,2.68]$} & 20 & 10 & 20 & {$[0: 0.5: 1.5] \pi$} \\
\hline Case 2 & 2.68 & 0.1 & {$[0,2.68]$} & 25 & 5 & 20 & {$[0: 0.5: 1.5] \pi$} \\
\hline \hline
\end{tabular}

To better understand the motion of the airfoil and flap with different flap phases, Figure 5 graphically depicts the kinematic motion along with airfoil/flap positions for case $1 \mathrm{a}$ or $1 \mathrm{~b}$ where $\alpha_{\text {mean }}=0^{\circ}$. The four different flap phases 
are plotted along with the airfoil motion schematics at four instances in the cycle. The arrows on the airfoil motion schematic show the direction of motion of the airfoil and flap.

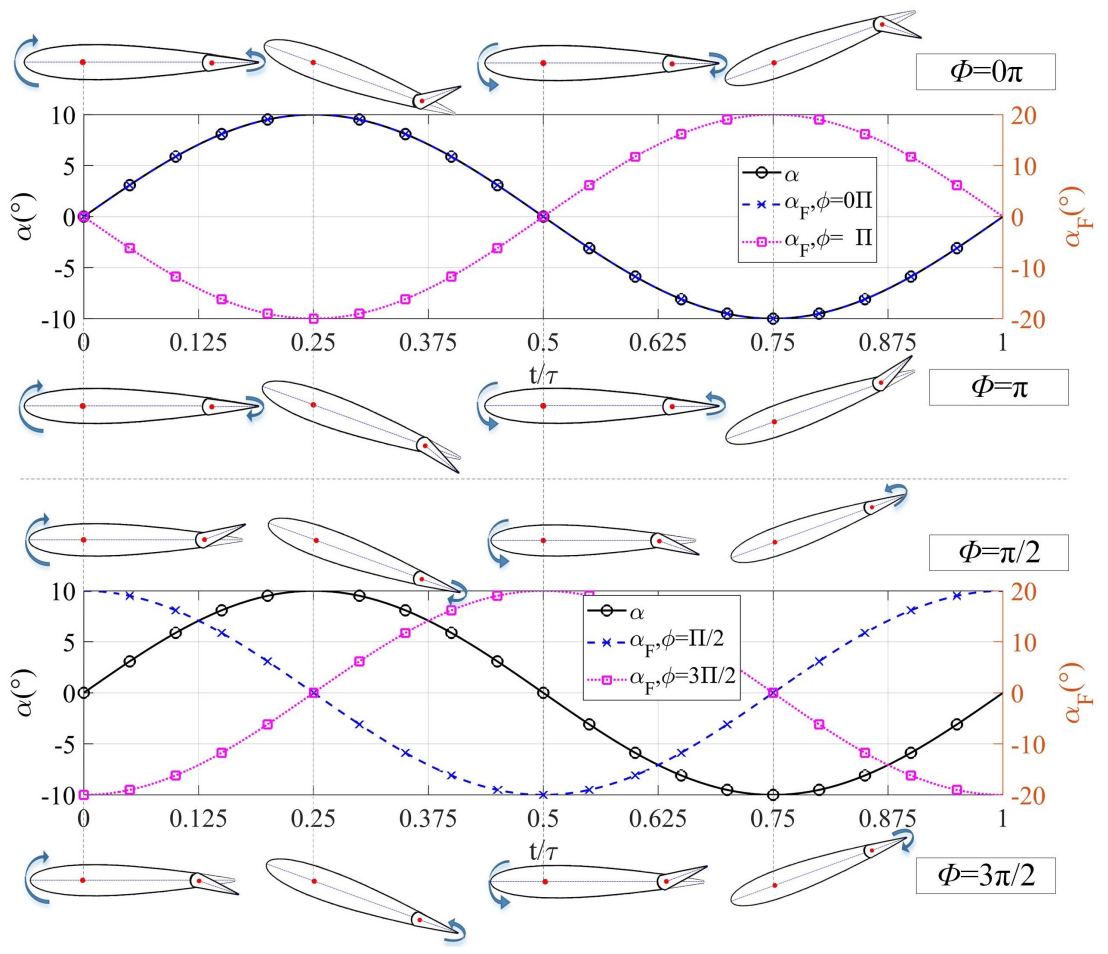

Figure 5. Kinematics of the airfoil and TEF motion for $\alpha_{\text {mean }}=0^{\circ}$ and different $\phi$ for case $1 \mathrm{a}$ or $1 \mathrm{~b}$

\section{Phase-Averaged Cycle Convergence Study}

A convergence study was undertaken to ensure the phase-averaged data presented is repeatable, reliable and the number of cycles used is sufficient to represent the averaged cycle-to-cycle variation due to the unsteady nature of the flow. The data presented in Figure 6 show the convergence study on the phase-averaged $C_{L}-\alpha$ plot and its uncertainty for case $1 \mathrm{~b}$ where $k=0.1$ and the airfoil is undergoing deep stall. In this convergence study the phase-averaged data is presented for $5,10,50$, 100,200 , and 500 cycles. The loop for 5 cycles is only presented to show that even at low cycle number the results are representative of the converged results. Figure 6a also shows repeatability and high confidence in the results and the phase-averaging procedure used to create the plots presented in this paper. The hysteresis loops in Figure 6a start to converge at 100 cycles within 1\%. This shows that the use of 200 cycles is sufficient to accurately determine the phase-averaged data for $C_{L}$. The $C_{L}$ uncertainty presented in Figure $6 \mathrm{~b}$ is the root sum squared of the bias and precision error. Bias error, also known as fixed or systematic error, is mainly due to hysteresis, linearity and temperature shift in the pressure transducers all of which tend to be constant from cycle-to-cycle. Precision error, on the other hand, is dependent on the cycle-to-cycle variation and the number of cycles collected. The two uncertainty in $C_{L}$ peaks in Figure $6 \mathrm{~b}$ are then not due to the bias error (because it is constant from cycle-to-cycle) but are due to the precision error as the two peaks represent the motion of the LEVs that tend to be dynamic and unsteady in nature. Most of the cycle-to-cycle variation is likely due to the uncontrolled differences in LEV formation and shedding. Figure $6 \mathrm{~b}$ also shows very low $C_{L}$ uncertainty values suggesting high repeatability of the complex flow occurring and that is worth noting. Based on the results presented in this section, at least 500 cycles were used to analyze and produce the subsequent plots. 


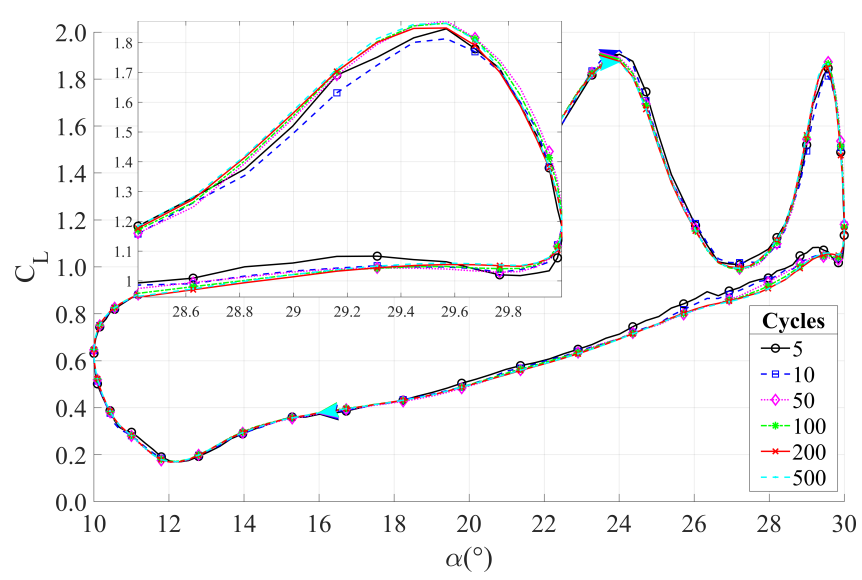

(a) Coefficient of lift

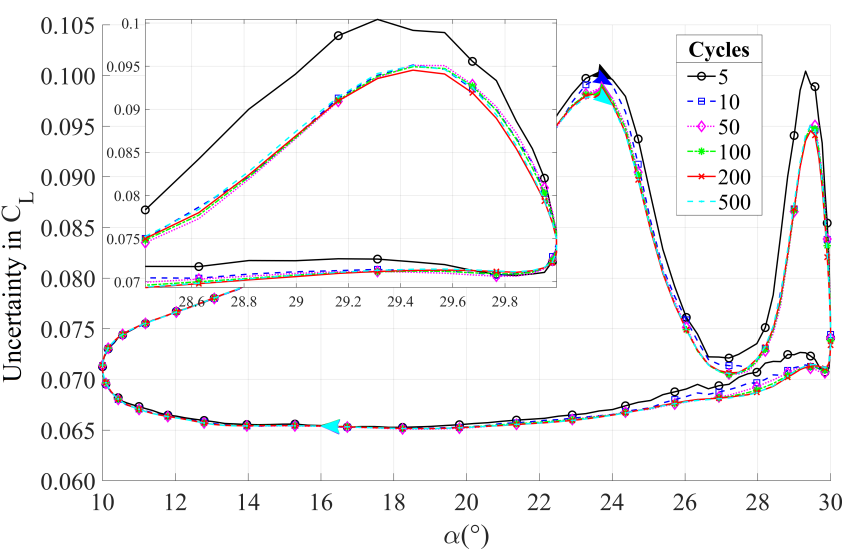

(b) Uncertainty in Lift

Figure 6. A convergence study of the phase-averaged data for different numbers of cycles for a) $C_{L}-\alpha$ b) Uncertainty in $C_{L}$ where $k=0.1$ and $\alpha_{\text {mean }}=20^{\circ}$ while the flap was fixed. An inset is used to better show the differences between $\alpha=28.4^{\circ}$ and $30^{\circ}$.

\section{Results}

Results include a brief description on how large flap angles could influence the aerodynamics of the airfoil by studying the static $C_{L}-\alpha, C_{M}-\alpha$ and $\Delta C_{p}$. The second section studies the hysteresis loop while the airfoil is dynamically pitching at two different $k$ values and two $\alpha_{\text {mean }}$ angles.

\section{Static Airfoil Characterization}

Instead of typically plotting $C_{L}$ or $C_{M}$ versus $\alpha$ for the static cases, they are plotted against $\alpha_{F}$ in Figure 7 . Having $\alpha_{F}$ on the x-axis clearly shows how the TEF influences the lift and moment for a constant $\alpha$. The static flap angle, $\alpha_{F}$, ranged from $-40^{\circ}$ to $60^{\circ}$ in steps of $10^{\circ}$ and the pitch angle, $\alpha$ ranged from $0^{\circ}$ to $25^{\circ}$ in steps of $5^{\circ}$. A more typical lift data versus $\alpha$ for different flap angles could be found in Samara and Johnson (2020a) for the same airfoil. To better understand the $C_{L}$ data it is important to simultaneously study the contour plots in Figure 8 where $\Delta C_{p}$ is plotted against the chord on the $\mathrm{x}$-axis and $\alpha$ on the y-axis ranging from $-40^{\circ}$ to $60^{\circ}$. Six individual $\alpha$ cases are plotted ranging from $0^{\circ}$ to $25^{\circ}$ in steps of $5^{\circ}$. Each contour plot is a matrix of 27 pressure measurements by $11 \alpha_{F}$ angles and each point is the average of 4000 data points over a period of 4 seconds. It is imperative then to draw conclusions from both figures (Figures 7 and 8) simultaneously as they complement each other.

Focusing on Figure 7a, as $\alpha_{F}$ increases (decreasing airfoil camber), $C_{L}$ decreases as long as $\alpha \leq 10^{\circ}$ where the flow is attached. When $\alpha=15^{\circ}$ the airfoil is operating at the onset of stall but complete flow separation is influenced by $\alpha_{F}$ because $C_{L}$ is higher than the $\alpha=10^{\circ}$ case for three $\alpha_{F}$ values and lower for the others. In Figure $8 \mathrm{~d}\left(\alpha=15^{\circ}\right)$ when $\alpha_{F}=-40^{\circ}$ or $-30^{\circ}$, the flow over the airfoil is completely stalled reducing $C_{L}$ because the $-\Delta C_{p}$ peak at the LE is greatly diminished. When $-20 \leq \alpha_{F} \leq 20^{\circ}$ the flow is partially attached as evident by the high $-\Delta C_{p}$ concentration at the LE but not completely attached because $C_{L}$ is lower than when $\alpha=10^{\circ}$. Lastly, for $\alpha_{F} \geq 30^{\circ}$ the flow is completely attached as evident by an increase in $-\Delta C_{p}$ at $\mathrm{x} / \mathrm{c}=0.3$ and $C_{L}$ is higher than when $\alpha=10^{\circ}$. For $\alpha=20^{\circ}$ and $25^{\circ}$, the trends are similar and could be explained in a similar way. For $\alpha_{F} \leq 20^{\circ}$ the flow is completely separated because $C_{L}$ is reduced and the $-\Delta C_{p}$ peak at the LE is also greatly diminished as seen in Figures $8 \mathrm{e}$ and 8f. When $\alpha_{F}>20^{\circ} C_{L}$ increases instead of decreasing as $\alpha_{F}$ increases. This could be attributed to the fact that as the flap angle becomes more positive the flow starts to partially reattach increasing $-\Delta C_{p}$ along the entire length of the chord as seen in the contour plots.

The TEF can significantly influence $C_{L}$ as long as the airfoil is not stalled but even in stalled cases some influence is still present. For example, $C_{L}$ changed from 1.3 to 0 for $\alpha=10^{\circ}$ by just changing the flap angle. The slope of $C_{L}$ change $\left(d\left(C_{L}\right) / d\left(\alpha_{F}\right)\right)$ is then $0.13 / 10^{\circ}$ as long as the airfoil is not stalled. This linear slope is similar to what was found by Abbott and Doenhoff (1959). The TEF was capable of reattaching the flow over the airfoil for $\alpha=15^{\circ}$ and partially reattaching the flow for $\alpha=20^{\circ}$ and $25^{\circ}$. The TEF is also capable of producing much higher $C_{L}$ values than by just changing $\alpha$. This was also observed by Abbott and Greenberg (1939) for a different airfoil but they also found that $C_{D}$ increases significantly for positive flap angles only. The maximum $C_{L}$ value for any $\alpha$ for an airfoil 
with no TEF deflection $\left(\alpha_{F}=0^{\circ}\right)$ is 0.9 but the highest $C_{L}$ value with the TEF is 1.3 thus 0.4 higher than with no TEF. It is important to note that even though the airfoil is stalled $\left(\alpha=20^{\circ}\right.$ or $\left.25^{\circ}\right)$, the TEF still has significant impact on $C_{L}$.

In Figure 7b as $\alpha_{F}$ increases (decreasing airfoil camber) $C_{M}$ increases except for select cases. From the figure it is seen that the flap angle has a large impact on the $C_{M}$ values. This could be better explained by investigating the $\Delta C_{p}$ contour plots in Figure 8. The contour plots show that as $\alpha_{F}$ changes, the center of pressure moves along the airfoil chord. $C_{M}$ tends to be more sensitive to the pressure center location than the magnitude of pressure on the airfoil surface. For example when $\Delta C_{p}$ is concentrated at the LE this causes the airfoil to pitch nose-up making $C_{M}$ positive. In contrast, when $\Delta C_{p}$ is concentrated at the TE that causes the airfoil to pitch nose-down making $C_{M}$ negative. Typically for non-stalled airfoils, positive $\alpha_{F}$ values decrease $-\Delta C_{p}$ at the TE and the airfoil is in pitch nose-up leading to a positive $C_{M}$. The opposite is true, negative $\alpha_{F}$ values increase $-\Delta C_{p}$ at the TE and the airfoil is in pitch nose-down leading to a negative $C_{M}$. When $\alpha=20^{\circ}$ and $25^{\circ}$, the slope in the $C_{M}$ data changes at $\alpha_{F}=20^{\circ}$ because $-\Delta C_{p}$ increases close to the TE as seen in the contour plots and this is attributed to partial flow reattachment. Changing $\alpha_{F}$ has a larger impact on $C_{M}$ when compared to the change in $\alpha$ because the center of pressure does not change with $\alpha$ as was also found by Abbott and Doenhoff (1959). In conclusion the TEF influences the flow dynamics over the airfoil with or without stall manipulating $C_{L}$ and $C_{M}$.

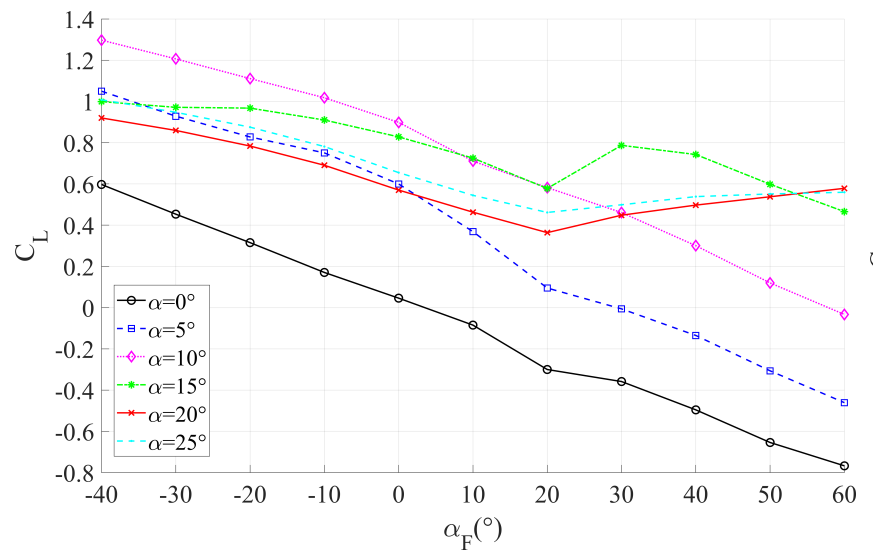

(a) Coefficient of lift versus $\alpha_{F}$

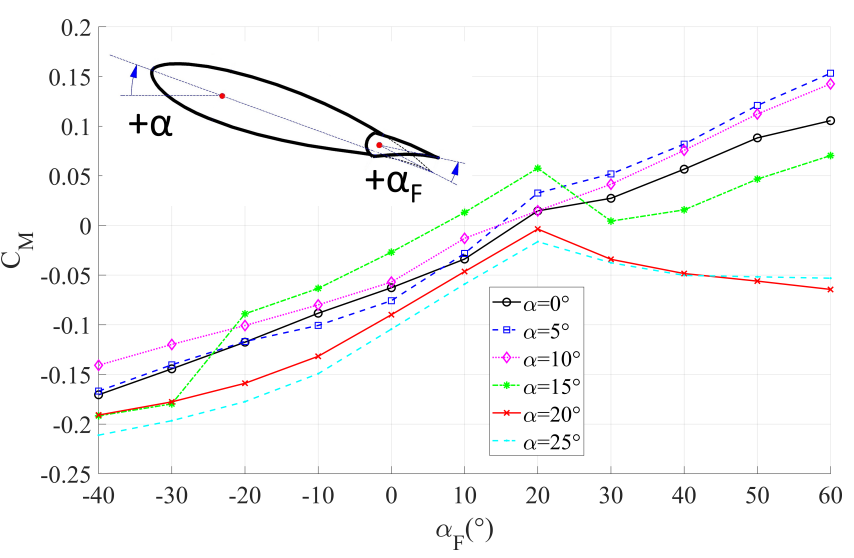

(b) Coefficient of moment versus $\alpha_{F}$

Figure 7. Measured coefficient of lift $\left(C_{L}\right)$ and moment $\left(C_{M}\right)$ versus $\alpha_{F}$ for several $\alpha$ values

In Figures 8a-c the TEF not only changes $\Delta C_{p}$ close to the TE but also at the LE as well. This indicates how the TEF influences the airflow over the entire chord and not just where the flap is located. In Figure 8d it can be seen that the flow is separated for $\alpha_{F}=-40^{\circ}$ and $-30^{\circ}$ because the $-\Delta C_{p}$ peak at the LE is absent. Looking at the same plot, at $\alpha_{F}=30^{\circ}$ there is an increase in $-\Delta C_{p}$ close to the LE and that increased the $C_{L}$ value in Figure 7a. In Figures $8 \mathrm{e}-\mathrm{f}$ the trend is similar in both cases and the flow is separated for all $\alpha_{F}$. Once the airfoil is stalled then changing the flap angle will not have significant impact on $\Delta C_{p}$ close to the LE but still could impact $\Delta C_{p}$ in the TE region. The isobars in all the plots help show how the TEF impacts $\Delta C_{p}$. The isobars of $\Delta C_{p}=0$ is marked in white dashes to better show negative $-\Delta C_{p}$ regions. The data and patterns in the contour plots are similar to what was found by Jacobs and Pinkerton (1931) on a RAF 30 airfoil.

\section{Dynamic Pitching}

In this section the dynamic pitching and flapping results are presented. The motion of the airfoil and flap are governed by Equations (1) and (2) and the parameters are presented in Table 1. A discussion and detailed analysis regarding the two different cases where $\alpha_{\text {mean }}$ is $20^{\circ}$ and $25^{\circ}$ are presented. The reason these cases were chosen is explained later in the discussion.

$\alpha_{\text {mean }}=20^{\circ}$

The data presented in Figures 9 to 14 belong to cases $1 \mathrm{a}$ and $1 \mathrm{~b}$ where $k=0.06$ and 0.1 respectively with $\alpha_{\text {mean }}=20^{\circ}$ and $\alpha_{a m p}=10^{\circ}$. The latter parameters were chosen so the airfoil is operating in deep dynamic stall and around the static stall angle so the flow has the opportunity to reattach on the airfoil. This way the airfoil is oscillating around 

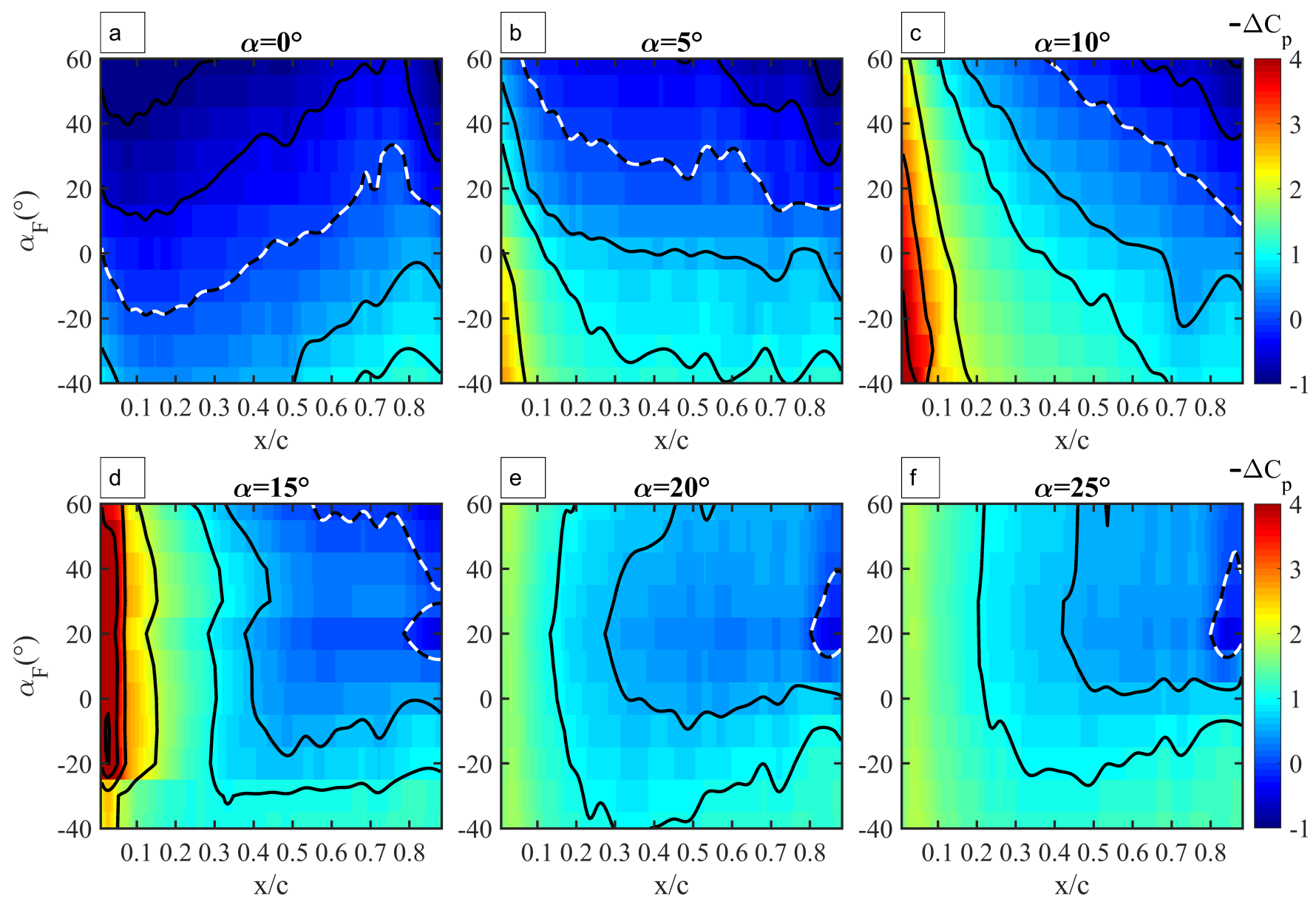

Figure 8. Contours of $-\Delta C_{p}$ versus $\mathrm{x} / \mathrm{c}$ versus $\alpha_{F}$. a) $\alpha=0^{\circ}$, b) $\alpha=5^{\circ}$, c) $\alpha=10^{\circ}$, d) $\alpha=15^{\circ}$, e) $\alpha=20^{\circ}$ and f) $\alpha=25^{\circ}$. The isobars on the plot represent constant $-\Delta C_{p}$ values $=[-1,-0.5,0,0.5,1,2,3,4]$. The isobars of $\Delta C_{p}=0$ is marked in white dashes.

the static stall angle which is around $16^{\circ}$. Approximately 600 cycles were collected for each scenario and each cycle was binned into 160 equal bins based on cycle period. $C_{L}$ and $C_{M}$ are presented in Figure 9 against $\alpha$ for the phase-averaged cycle. The arrows on the plot indicate the direction of motion. The "FixedFlap" case represents a pitching airfoil where the flap is not moving relative to the airfoil. Four equally spaced phase shifts between the pitch and the flap motion denoted by $\phi$ in Equation (2) are presented.

The $C_{L}$ phase-averaged loops are presented in two plots, Figures 9a and 9c, where each plot contains a FixedFlap loop, static airfoil data, and 2 flap phases (first: $\phi=0 \pi$ and $\pi / 2$, second: $\phi=\pi$ and $3 \pi / 2$ ). The static lift data (red stars) is added for comparison. During dynamic stall, $C_{L}$ is much higher than the static lift data and at certain pitch angles more than twice as much. A similar trend could be seen across the different flap phases but with a slightly different magnitude and location. Starting at $\alpha=10^{\circ}$ as the airfoil pitches up, $C_{L}$ starts to increase but then around $\alpha=21^{\circ}$ there is a sudden increase in $C_{L}$ caused by the primary LEV. After the LEV convects off the airfoil, the flow is separated. Then around $\alpha=26^{\circ}$ there is a second sharp increase in $C_{L}$ this time it is due to a secondary LEV which tends to be weaker in magnitude. The secondary LEV is followed by a subsequent small LEV before the downstroke portion of the cycle. The formation of multiple LEVs is in agreement with past experimental studies and numerical models (Leishman, 1990; Gharali and Johnson, 2013; Zanotti and Gibertini, 2013). It is interesting to note that all the LEV occur during the upstroke only as seen by McCroskey (1981). This could be attributed to the low $k$ value of 0.06 and high $\alpha_{\text {mean }}$ of $20^{\circ}$. During downstroke, the airfoil is stalled and $C_{L}$ keeps decreasing until around $\alpha=14^{\circ}$ where the flow starts to reattach and $C_{L}$ starts to increase. It is interesting to note that $C_{L}$ is higher than the static points even during the downstroke cycle where the airfoil is stalled and this is due to the nature of dynamic stall. Comparing $\phi=3 \pi / 2$ to the FixedFlap, the primary and secondary LEV (which is seen by a spike in 
the $C_{L}-\alpha$ plots) occur $1^{\circ}$ earlier while for $\phi=\pi / 2$ the LEV occurs $1^{\circ}$ later. In the other two $\phi$ cases $(0 \pi$ and $\pi)$, the LEV location does not change. Out of all the different $\phi$ tested it could be concluded that when $\phi=\pi / 2, C_{L}$ has the least fluctuation. This is because most of the high peak loads occur halfway through the upstroke portion of the cycle where $\alpha=20^{\circ}$. For $\phi=\pi / 2$, the magnitude of the $C_{L}$ peaks caused by the LEV are smaller than the other cases and the hysteresis cycle is narrow. To better understand the kinematics of the pitch and flap motion and the phase offset between the two, refer to Figure 5.

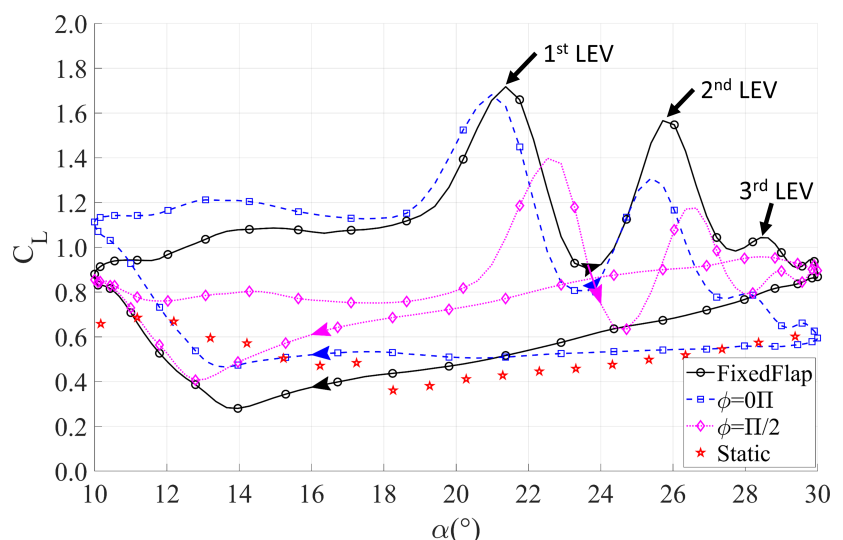

(a) Coefficient of lift $(\phi=0 \pi, \phi=\pi / 2)$

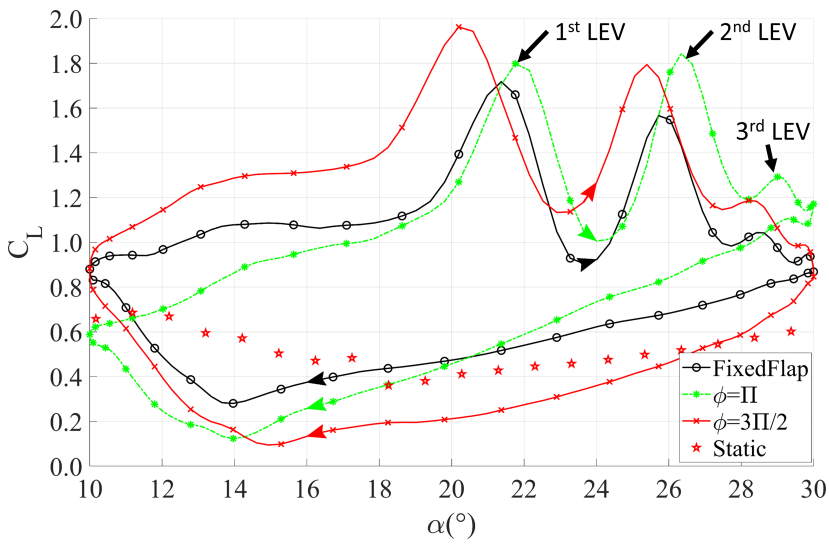

(c) Coefficient of lift $(\phi=1 \pi, \phi=3 \pi / 2)$

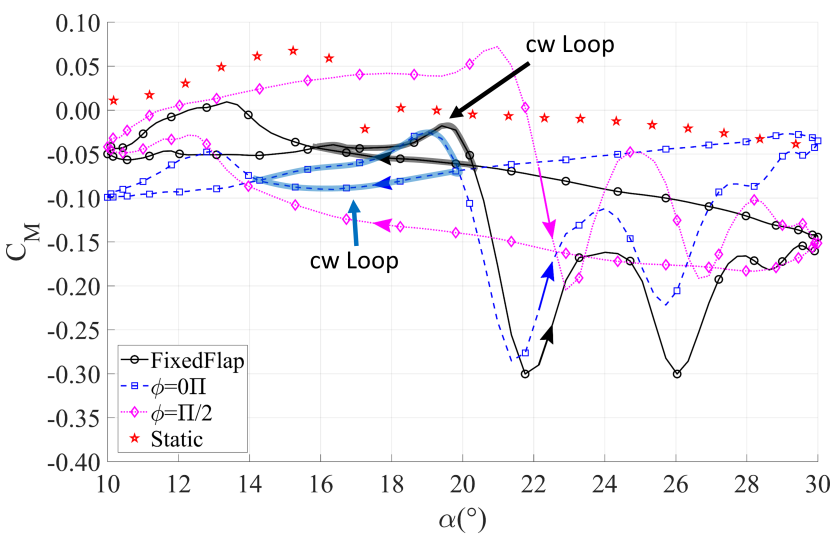

(b) Coefficient of moment $(\phi=0 \pi, \phi=\pi / 2)$

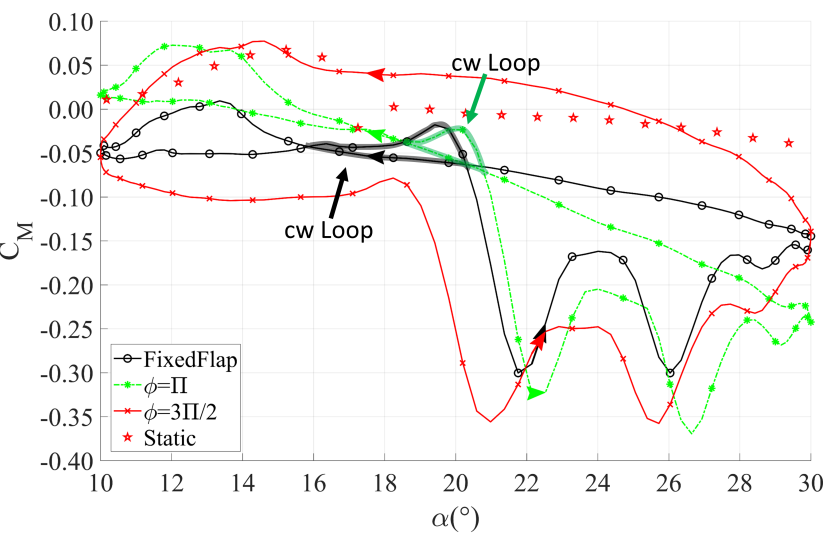

(d) Coefficient of moment $(\phi=1 \pi, \phi=3 \pi / 2)$

Figure 9. Coefficient of lift $\left(C_{L}\right)$, and moment $\left(C_{M}\right)$ versus $\alpha$ for different flap phases $(\phi)$ for case 1a where $k=0.06$ and $\alpha_{\text {mean }}=20^{\circ}$. Only every $4^{t h}$ data point is shown for clarity. The $1^{\text {st }}$ and $2^{\text {nd }}$ LEV and the cw loops are marked in each plot.

Figures $9 \mathrm{~b}$ and $9 \mathrm{~d}$ show the $C_{M}$ hysteresis loops for case 1a. During dynamic stall the LEV starts from the LE and sweeps towards the TE. While the vortex sweeps over the airfoil, it produces a rapid aft movement of the center of pressure from the LE towards the TE resulting in a large nose-down pitching moment on the airfoil (Leishman, 2006). When the LEV is at the LE it increases $C_{L}$ but when the LEV is shed towards the TE it increases the $C_{M}$ value. This is why the peak in $C_{M}$ always occurs after the peak in $C_{L}$ for all cases. $C_{M}$ is in constant nose-down pitching (negative $C_{M}$ value) for the different $\phi$ cases when compared to the static values. This indicates that the center of pressure is always behind the quarter-chord of the airfoil towards the TE. Another important point to notice in the $C_{M}$ loop is the formation of a clockwise (cw) loop that indicates negative damping. A small cw loop is found in the FixedFlap, $\phi=0 \pi$, and $\phi=\pi$ around $\alpha=18^{\circ}$. For $\phi=\pi / 2$, the entire loop is cw promoting stall flutter while the entire loop for $\phi=3 \pi / 2$ is counter-clockwise (ccw) preventing stall flutter at all times. As discussed in the introduction, negative damping promotes flutter and instabilities that lead to severe vibration and mechanical failure if the structural dynamics permit and would be best avoided at all times (Corke and Thomas, 2015). In this experimental setup when negative damping was present, the airfoil section was observed to experience excessive vibration. 
Figure 10 represents $C_{L}$ and $C_{M}$ for case $1 \mathrm{~b}$ where $k$ increased from 0.06 to 0.1 and $\alpha_{\text {mean }}=20^{\circ}$. Focusing on the $C_{L}$ data there are few major differences when $k$ increases. First, the amplitude of the $C_{L}$ peaks caused by the LEV are increased and the occurrence of the LEV is delayed by $3^{\circ}$. The primary LEV occurs around $24^{\circ}$ followed by a secondary LEV at $29^{\circ}$. This delay in occurrence of the LEV is mainly due to the increase in $k$ that tends to delay dynamic stall to a higher $\alpha$ value (Corke and Thomas, 2015; Leishman, 1990). The secondary LEV again tends to be weaker than the primary LEV. There is also a very small subsequent LEV occurring during the first part of the downstroke cycle. The difference between the upstroke and downstroke increases, widening the hysteresis loop and increasing cyclic loading when $k$ increases. These differences mentioned are in agreement with prior studies (Leishman, 2006; Lee and Gerontakos, 2004; McCroskey, 1981). The loss of lift after the secondary LEV is much steeper and sudden when compared to the $k=0.06$ case because the secondary LEV occurs when the airfoil is about to change pitch direction at $\alpha=30^{\circ}$. From cases 1a and 1b, it is concluded from the $C_{L}$ loops that for $\phi=\pi / 2$ the load fluctuation is smallest, the hysteresis loops are much narrower and the peaks due to the LEV are less severe. On the other hand, $\phi=3 \pi / 2$ has the largest load fluctuations, the widest hysteresis loops and the highest peaks.

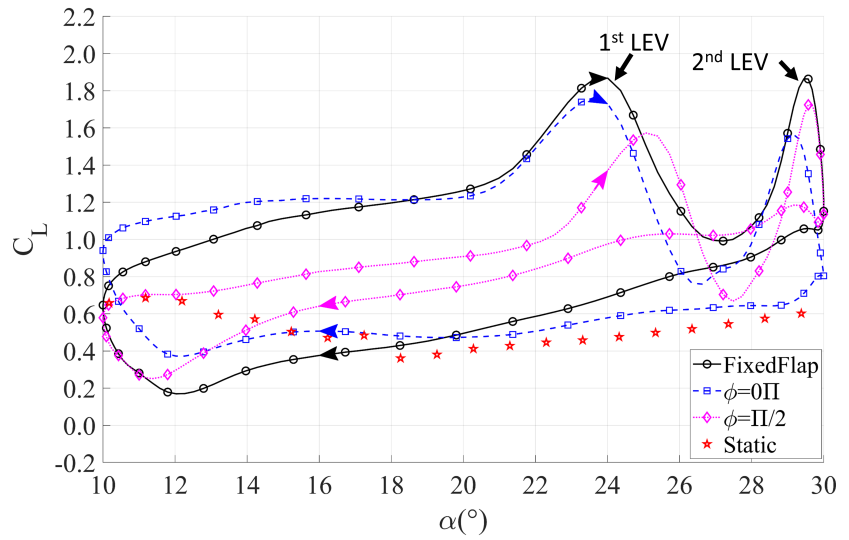

(a) Coefficient of lift $(\phi=0 \pi, \phi=\pi / 2)$

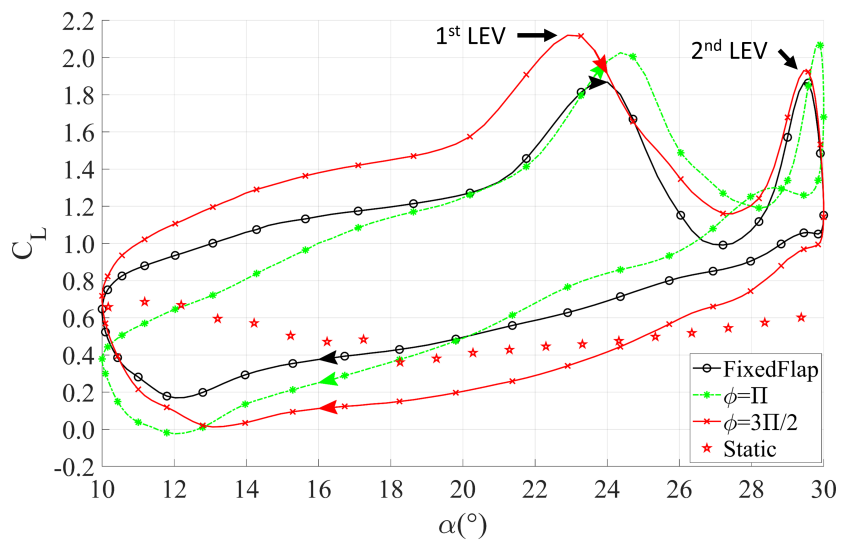

(c) Coefficient of lift $(\phi=1 \pi, \phi=3 \pi / 2)$

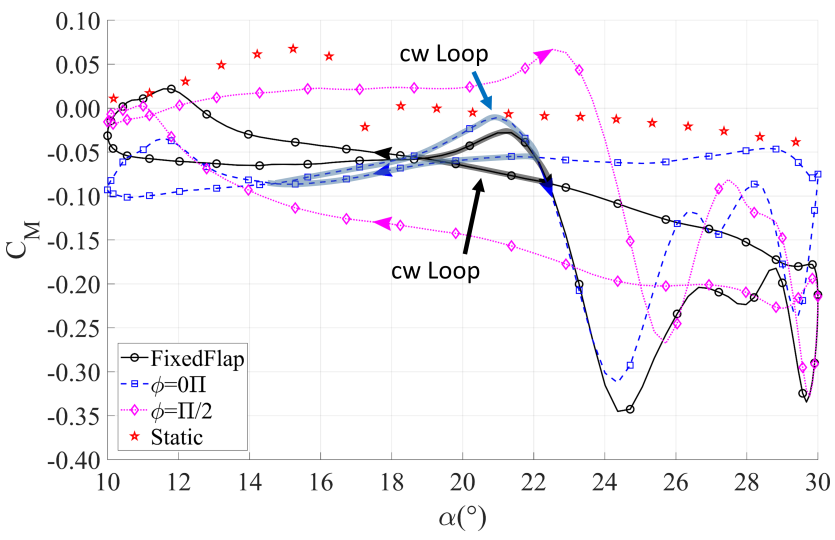

(b) Coefficient of moment $(\phi=0 \pi, \phi=\pi / 2)$

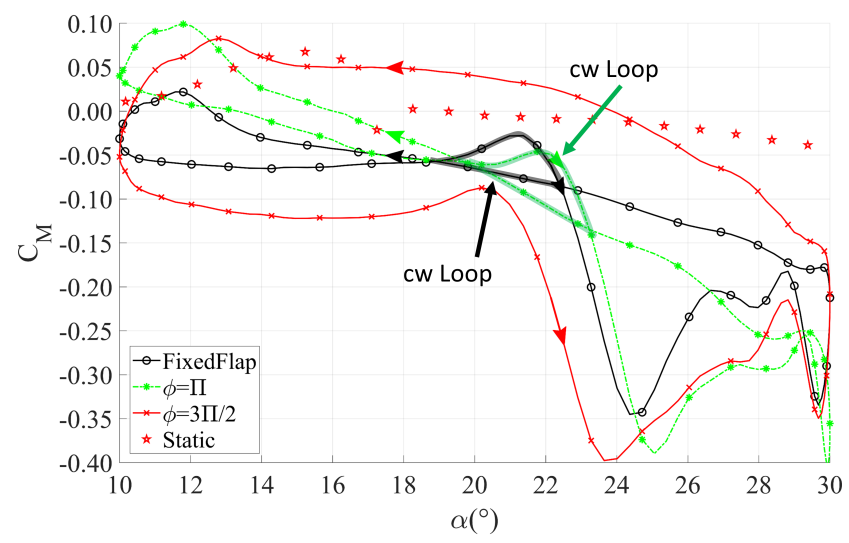

(d) Coefficient of moment $(\phi=1 \pi, \phi=3 \pi / 2)$

Figure 10. Coefficient of lift $\left(C_{L}\right)$, and moment $\left(C_{M}\right)$ versus $\alpha$ for different flap phases $(\phi)$ for case $1 \mathrm{~b}$ where $k=0.1$ and $\alpha_{\text {mean }}=20^{\circ}$. Only every $4^{\text {th }}$ data point is shown for clarity. The $1^{\text {st }}$ and $2^{\text {nd }}$ LEV and the cw loops are marked in each plot.

Looking at the $C_{M}$ hysteresis plots in Figure 10 and comparing them to Figure 9 where $k$ increased from 0.06 to 0.1 a few differences arise. $C_{M}$ peaks are increased and the LEV formation is delayed as seen in the $C_{L}$ plots. The cw loops now occur around $\alpha=21^{\circ}$ instead of $18^{\circ}$. The magnitude of negative damping is about the same. A similar conclusion to that made for the $C_{L}$ loops is made for the $C_{M}$ loops, $\phi=\pi / 2$ produces the least fluctuations and $\phi=3 \pi / 2$ produces the highest fluctuations. On the other hand, $\phi=\pi / 2$ leads to a negatively damped $C_{M}$ loop promoting flutter while $\phi=3 \pi / 2$ leads to positive damping. To conclude which $\phi$ to use to minimize dynamic stall on the airfoil, $\phi=\pi / 2$ greatly reduces $C_{L}$ but at the same time leads to negative damping. Another option is $\phi=0 \pi$ 
and that reduces $C_{L}$ peaks slightly but eliminates negative damping. So depending whether to reduce $C_{L}$ peaks or eliminate negative damping, $\phi$ should be $\pi / 2$ or $0 \pi$.

Plotting $C_{L}$ and $C_{M}$ versus $\alpha$ is helpful to see the overall trends in the data. To better understand how the pressure center and magnitude changes over the airfoil surface, a contour plot is used to plot $\Delta C_{p}$ versus airfoil chord on the $\mathrm{x}$-axis $(\mathrm{x} / \mathrm{c})$ versus averaged cycle period, $t / \tau$ (where $t$ is time and $\tau$ is the motion cycle period), on the y-axis. Arrows along the y-axis indicate the direction of airfoil motion, whether it would be upstroke or downstroke. The cycle period, $t / \tau$, could also be associated with $\alpha$ as shown on the right $y$-axis and the relationship is plotted in Figure $11 \mathrm{~d}$ for $\phi=0 \pi$. The plots are constructed from a matrix of 27 differential pressure measurements along the airfoil chord by 160 bins representing one complete cycle. Each point in the matrix is the average of 4000 data points. Figure 11 shows the $\Delta C_{p}$ contour plots for case $1 \mathrm{~b}$ for FixedFlap, $\phi=\pi / 2$, and $\phi=3 \pi / 2$ cases. The contour plots for case 1a are not be presented because the plots are very similar to those of case 1b. The pattern for all three $\phi$ cases presented is similar other than small magnitude and location differences and could be described at the same time. Starting at $\mathrm{t} / \tau=0,-\Delta C_{p}$ is concentrated at the $\mathrm{LE}(\mathrm{x} / \mathrm{c}<0.25)$ and starts to increase until it reaches its highest point at $\mathrm{t} / \tau \approx 0.25$ indicating that the flow is attached. After this point the LEV starts to shed from the LE to the TE. As the LEV is shed it tends to decrease $-\Delta C_{p}$ close to the LE but increases $-\Delta C_{p}$ on the rest of the airfoil surface. This increase in $-\Delta C_{p}$ over the airfoil creates the sudden peak in $C_{L}$ and $C_{M}$ as seen in Figures 9 and 10. After the primary LEV,$-\Delta C_{p}$ along the entire airfoil chord is diminished indicating stall until the secondary LEV is formed at $\mathrm{t} / \tau \approx 0.45$ but with a weaker magnitude. After the secondary LEV, $-\Delta C_{p}$ along the entire airfoil chord is diminished again for the rest of the cycle indicating stall. This phase-averaged pattern in $\Delta C_{p}$ for the pitching cycle and how it shows both LEV was also seen experimentally by Zanotti (2012) and Zanotti and Gibertini (2013) for a NACA 23012 airfoil without a TEF. Comparing the three different $\phi$ cases, the magnitude of the LEV is increased for $\phi=3 \pi / 2$ and decreased for the $\phi=\pi / 2$ when compared to the FixedFlap as seen more clearly through the isobars on the plots. This comparison was also made by Lee and $S u(2011)$ from the time history of $\Delta C_{p}$. This concludes that the TEF is not capable of eliminating the LEV formation that causes spikes in the $C_{L}-\alpha$ and $\Delta C_{p}$ plots but it could reduce its impact on the airfoil.

A different approach to visualize the airfoil stall and the vortex structures can be seen by looking at the standard deviation (STD) in $\Delta C_{p}$ which is plotted in Figure 12 for case 1b. The STD is plotted in log scale to show the small changes at lower values. Stall and LEVs are typically associated with high pressure fluctuations because of the flow dynamics as reported by Leishman (2006) and these could also be seen in Figure 6 which shows the largest fluctuations occur when the LEV detaches. While $0<\mathrm{t} / \tau<0.25$ and for all the cases presented, $\operatorname{STD}\left(\Delta C_{p}\right)$ values are low indicating that the airfoil is not stalled. The primary and secondary LEV discussed in Figure 11 also appear in this figure and are associated with very high STD values at $\mathrm{t} / \tau \approx 0.25$ and $\mathrm{t} / \tau \approx 0.45$. The time history of the $\operatorname{STD}\left(\Delta C_{p}\right)$ clearly shows that the movement of the LEV is from the LE to the TE. The observations made here reinforce the discussion made earlier while discussing $C_{L}-\alpha$ and $\Delta C_{p}$ plots. After both vortices flow past the TE, STD is reduced but still much higher than at $\mathrm{t} / \tau=0$ indicating that the flow over the airfoil is stalled between $\mathrm{t} / \tau=0.5$ and 1 . The STD is then reduced further at $\mathrm{t} / \tau=0$ when the airfoil starts to pitch upwards indicating flow reattachment. This statement is confirmed in the $C_{L}-\alpha$ plot in Figure 10 showing that during the downstroke cycle, $C_{L}$ is much lower than the upstroke cycle until the very end of the cycle. The $\operatorname{STD}\left(\Delta C_{p}\right)$ patterns do not change significantly between the four $\phi$ cases presented in the figure other than a slight change in magnitude and location of the peaks.

So far $C_{L}-\alpha, C_{M}-\alpha, \Delta C_{p}$, and $\operatorname{STD}\left(\Delta C_{p}\right)$ have been discussed showing the complexity and behavior of dynamic stall but they do not clearly show with absolute certainty which $\phi$ case reduces the load fluctuations the most. To simply and effectively measure the impact of a TEF on an oscillating airfoil undergoing dynamic stall, a fast Fourier transform (FFT) was performed on the time resolved $C_{L}$ and root bending moment data separately. The frequency analysis plots are presented in Figure 13 for case 1a. The power spectral density (PSD) on the y-axis was normalized with respect to the peak amplitude found in the FixedFlap case. Frequency on the x-axis was normalized with respect to the oscillation frequency $\left(f_{p}\right)$ and thus the highest amplitude occurs at frequency $/ f_{p}=1$. There is another peak present at frequency $/ f_{p}=2$ representing the second harmonic of the oscillation. Both axes of the plot are in log scale to better show the frequency and amplitude distribution and an inset is used to magnify the results around $f_{p}$. The plots show that for $\phi=\pi / 2$, the $C_{L}$ peak amplitude at $f_{p}$ is reduced by $53 \%$ while the root bending moment amplitude is reduced by $37 \%$ with respect to the FixedFlap case. This reduction is significant and shows that the TEF is capable of reducing cyclic loading. There is a greater measured reduction in $C_{L}$ than root bending moment 

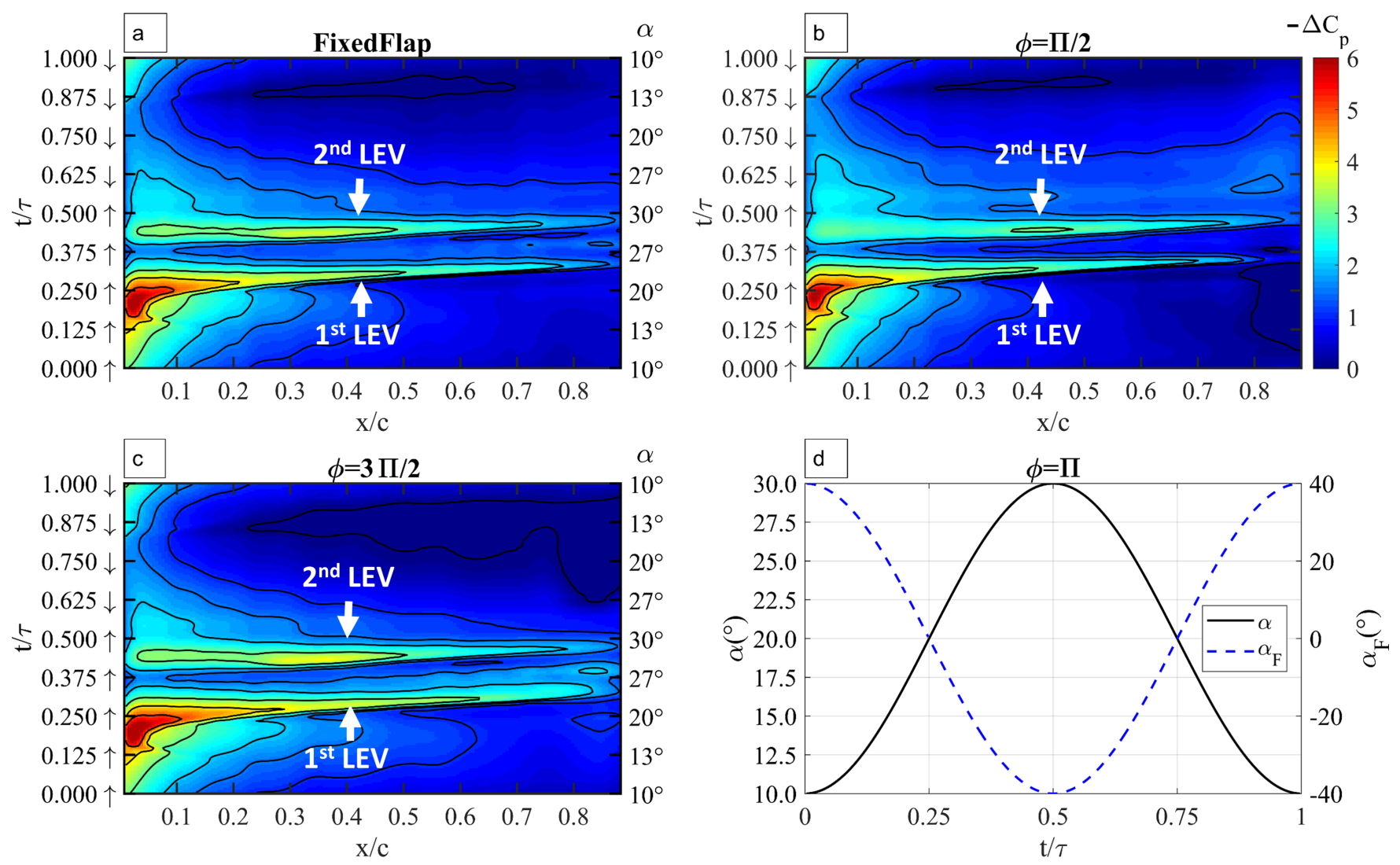

Figure 11. Contours of $-\Delta C_{p}$ versus $x / c$ versus $t / \tau$ for case $1 b\left(k=0.1\right.$ and $\left.\alpha_{\text {mean }}=20^{\circ}\right)$. a), b), and c) represent FixedFlap, $\phi=\pi / 2$, and $\phi=3 \pi / 2$ respectively. d) $\alpha$ versus pitch period $(\mathrm{t} / \tau)$, and flap angle $\left(\alpha_{F}\right)$ for phase $\phi=1 \pi$. The isobars on the plot represent constant $-\Delta C_{p}$ values $=[0,1,1.5,2,3,4,5]$.

because $C_{L}$ is measured at the center of the TEF section while the root bending moment measures the lift force for the entire airfoil section where the TEF occupies $60 \%$ of the airfoil section.

The PSD is also plotted for case $1 \mathrm{~b}$ in Figure 14. Comparing case $1 \mathrm{~b}$ to $1 \mathrm{a}$ and for $\phi=\pi / 2$, the $C_{L}$ amplitude is reduced by $26 \%$ compared to $53 \%$ while the root bending moment amplitude is reduced by $24 \%$ compared to $37 \%$. The change in $\%$ reduction occurs because higher $k$ values produce larger peaks in the $C_{L}-\alpha$ plots and the TEF influence over the latter variable is the same independent of $k$.

$\alpha_{\text {mean }}=25^{\circ}$

The results published by Gallant and Johnson (2017) indicate that when an operating wind turbine is yawed, the mean oscillating $\alpha$ could be $25^{\circ}$ while the amplitude could be $5^{\circ}$ under certain conditions. Case 2 in this paper aims to simulate a wind turbine blade under the same conditions mentioned in Gallant and Johnson. Figure 15 shows $C_{L}-\alpha$ and $C_{M}-\alpha$ for case 2 and for different $\phi$ cases where $k=0.1, \alpha_{\text {mean }}=25^{\circ}$, and $\alpha_{a m p}=5^{\circ}$. Focusing on the $C_{L}$ plots (Figures 15a and 15c), the FixedFlap cycle is cw and the hysteresis loop is narrow indicating that the difference between the upstroke and the downstroke motion is minimal. What is most interesting about the different loops is that the airfoil is stalled during the entire cycle but even so, $C_{L}$ is higher than its static counterpart. There are a few points that indicate that the airfoil is always stalled for all $\phi$ cases. First, the airfoil motion for case 2 prescribes $\alpha$ to range from $20^{\circ}$ to $30^{\circ}$ so $\alpha$ is never below the static stall angle thus reattachment does not occur. Second, the formation of a LEV is not present in any of the loops as there are no sudden peaks in the $C_{L}$ or $C_{M}$ plots. The lack of LEV formation indicates that the flow is always separated along the airfoil surface. Lastly the $\Delta C_{p}$ contour plots presented later in Figure 16 show that $-\Delta C_{p}$ is never concentrated at the LE and the values are smaller than the static case indicating flow separation. When comparing $\phi=\pi / 2$ or $3 \pi / 2$ to the FixedFlap loop it is found that the $C_{L}$ loop widens significantly but at the same time $C_{L}$ does not change 

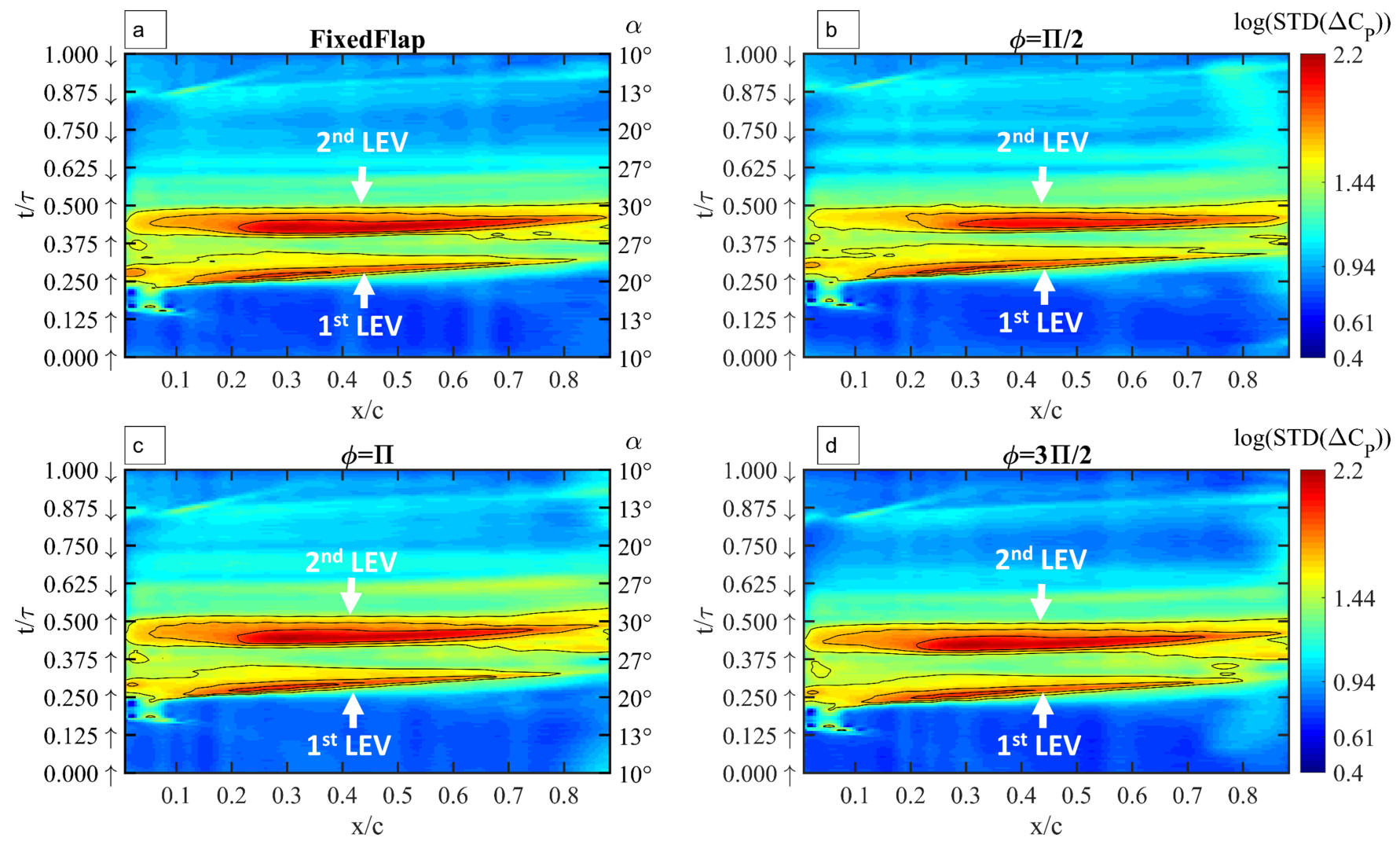

Figure 12. Contour plots showing the $\operatorname{STD}\left(\Delta C_{p}\right)$ versus $x / c$ versus $t / \tau$ for case $1 \mathrm{~b}$ where $k=0.1$ and $\alpha_{\text {mean }}=20^{\circ}$. a), b), c), and d) represent FixedFlap, $\phi=\pi / 2, \phi=1 \pi$, and $\phi=3 \pi / 2$ respectively. The isobars on the plot represent constant $\log \left(\operatorname{STD}\left(\Delta C_{p}\right)\right)$ values.

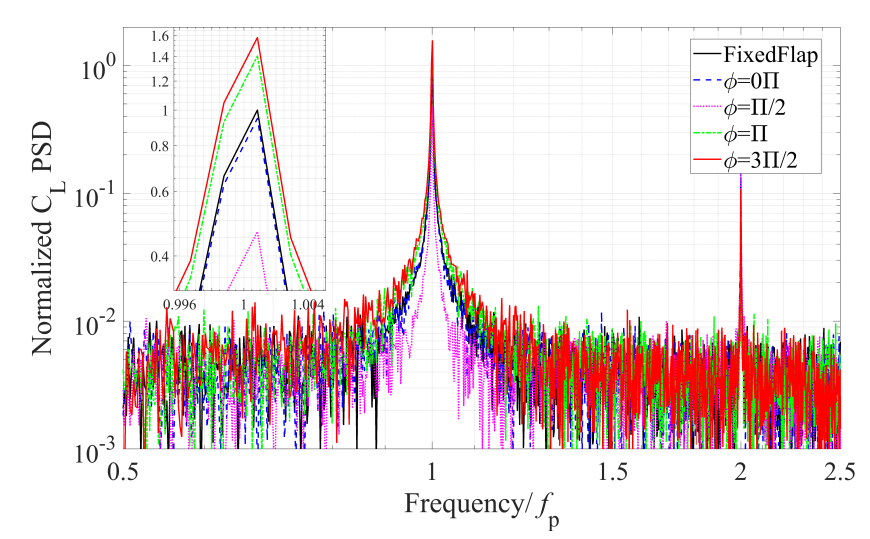

(a) Spectral density for $C_{L}$

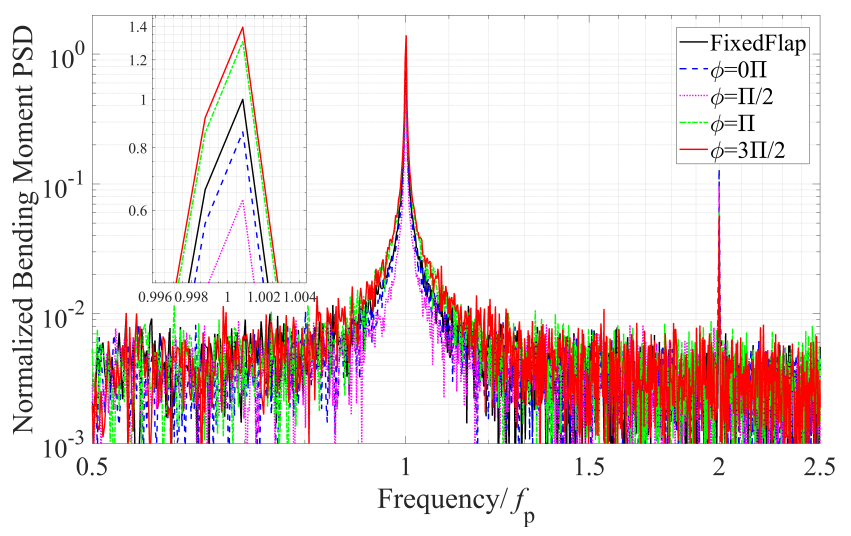

(b) Spectral density for root bending moment

Figure 13. Normalized power spectral density (PSD) distribution for $C_{L}$ and root bending moment for different flap phases ( $\left.\phi\right)$ for case $1 \mathrm{a}$ where $k=0.06$ and $\alpha_{\text {mean }}=20^{\circ}$. Frequency on the $\mathrm{x}$-axis was normalized with respect to the oscillation frequency $\left(f_{p}\right)$. Both axes are log scale and an inset is shown to magnify the results around $f_{p}$.

when $\alpha$ is at its extreme values. On the other hand, when comparing $\phi=0 \pi$ or $\phi=\pi$ to the FixedFlap loop, it was found that the width of the $C_{L}$ loops does not change and stays narrow but the $C_{L}$ extreme values are altered when $\alpha$ is at its minimum or maximum value. This shows that the TEF can manipulate $C_{L}$ at any point on the cycle and 


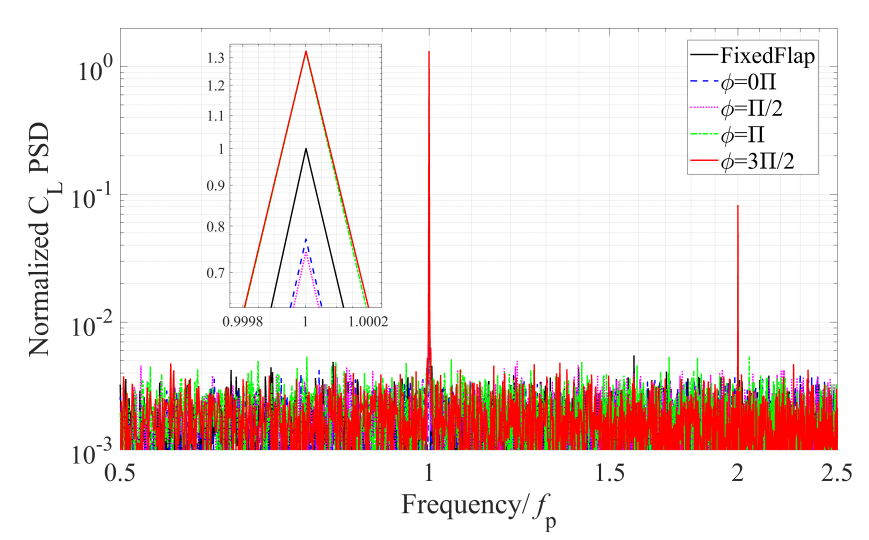

(a) Power spectral density for $C_{L}$

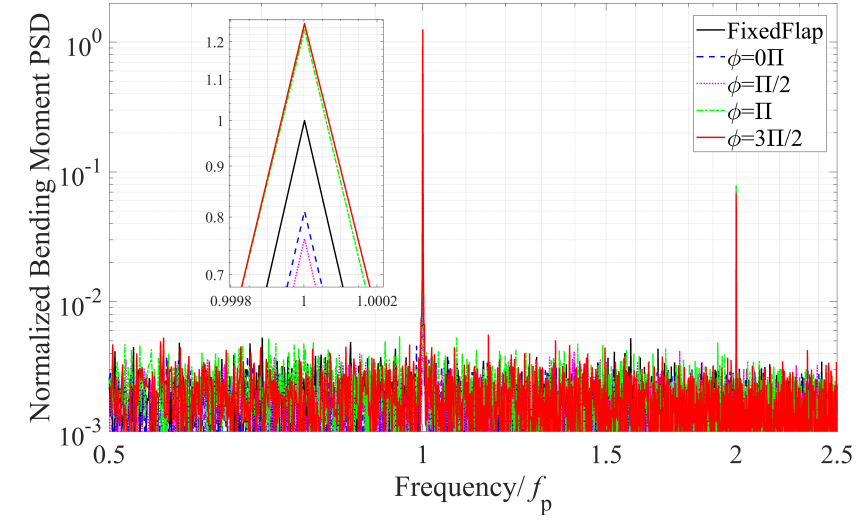

(b) Power spectral density for root bending moment

Figure 14. Normalized power spectral density (PSD) distribution for $C_{L}$, and root bending moment for different flap phases $(\phi)$ for case $1 \mathrm{~b}$ where $k=0.1$ and $\alpha_{\text {mean }}=20^{\circ}$. Frequency on the $\mathrm{x}$-axis was normalized with respect to the oscillation frequency $\left(f_{p}\right)$. Both axes are log scale and an inset is shown to magnify the results around $f_{p}$.

that point is determined by the variable $\phi$. To reduce the $C_{L}$ fluctuation and keep the value constant for the entire cycle then it is recommended to set $\phi=0 \pi$ and reduce $\alpha_{a m p, f}$ from $20^{\circ}$ to approximately $15^{\circ}$.

Figures $15 \mathrm{~b}$ and $15 \mathrm{~d}$ represent $C_{M}$ versus $\alpha$ for the different $\phi$ cases. For all cases, $C_{M}$ is lower than its static counterpart indicating the center of pressure is past the quarter-chord of the airfoil. This is confirmed by the $\Delta C_{p}$ contour plots in Figure 16. In the FixedFlap, $\phi=0 \pi$, and $\phi=\pi$ the $C_{M}$ loops are narrow and ccw eliminating the possibility of stall flutter. For $\phi=\pi / 2$ the $C_{M}$ loop widens significantly and is cw promoting stall flutter on the airfoil. As for $\phi=3 \pi / 2$, the $C_{M}$ loop is also wide but the loop is ccw eliminating the possibility of stall flutter. To reduce $C_{M}$ fluctuations and achieve a constant $C_{M}$, it is concluded to use $\phi=0 \pi$ but also reduce $\alpha_{a m p, f}$ from $20^{\circ}$ to approximately $15^{\circ}$. This conclusion is aligned with the one made for the $C_{M}-\alpha$ loops.

To study how the pressure over the airfoil surface changes in distribution and magnitude, $-\Delta C_{p}$ versus $\mathrm{x} / \mathrm{c}$ versus $\mathrm{t} / \tau$ is plotted in Figure 16 similar to the plots in Figure 11. From the contour plots, it could be noticed that the airfoil is stalled because the $-\Delta C_{p}$ values are much smaller and less concentrated at the LE when compared to case 1 in Figure 11. This occurs because for case 2, $\alpha$ never goes below the static angle and the flow does not have the opportunity to reattach over the airfoil surface. Case 2 is stalled during the entire cycle despite the fact that it is oscillating between $20^{\circ} \leq \alpha \leq 30^{\circ}$ and within the oscillating bound of case 1 where $10^{\circ} \leq \alpha \leq 30^{\circ}$ and the flow was attached during part of the case 1 cycle. When comparing $\phi=0 \pi$ to the FixedFlap case a few areas of interest are noted. At t $/ \tau=0.5,-\Delta C_{p}$ close to the TE $(\mathrm{x} / \mathrm{c}>0.75)$ becomes negative due to the fact that $\alpha_{F}=20^{\circ}$ is creating a lower suction pressure on the suction side. On the other hand, when $\mathrm{t} / \tau=0$ or 1 indicating that $\alpha_{F}=-20^{\circ}$, it creates a higher suction pressure on the suction side close to the TE. The opposite is true when $\phi=\pi$, the suction pressure increases at $\mathrm{t} / \tau=0.5$ and decreases at $\mathrm{t} / \tau=0$ and 1 when compared to the FixedFlap case. The TEF did not just have influence on the TE pressure but also on the LE pressure as well as could be seen in the contour plots and the isobars. This indicated that the TEF influences the aerodynamics around the entire airfoil when the flow is stalled or attached.

\section{Conclusions}

Initially static measurements of $C_{L}$ and $C_{M}$ versus large trailing edge flap (TEF) angles $\left(\alpha_{F}\right)$ were presented for an S833 airfoil installed with a TEF. Changing $\alpha_{F}$ is capable of producing much higher $C_{L}$ values than by just changing the pitch angle. The TEF can move the center of pressure on the airfoil surface thus greatly influencing $C_{M}$. The $\Delta C_{p}$ contour plots showed that the TEF influences the airflow over the entire chord and not just where the flap is located.

A series of hysteresis loops for $C_{L}$ and $C_{M}$ versus angle of attack $(\alpha)$ were presented for a pitching airfoil in deep dynamic stall with reduced frequencies $(k)$ of 0.06 and 0.1 . The TEF was oscillating at the same pitching frequency with a constant phase offset, $\phi$. It was found that $\phi$ has a strong impact on the hysteresis loops manipulating the load fluctuations. Due to dynamic stall, the pitching motion created two very noticeable leading edge vortices (LEVs) and 


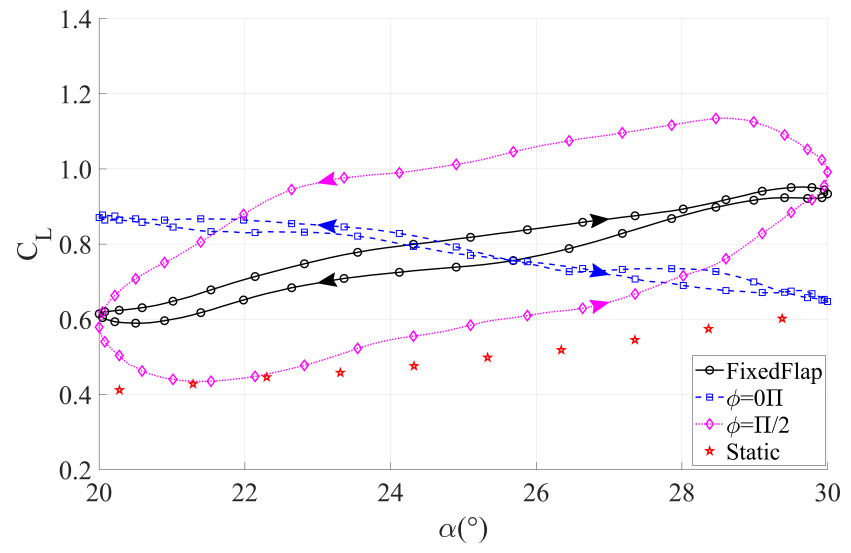

(a) Coefficient of lift $(\phi=0 \pi, \phi=\pi / 2)$

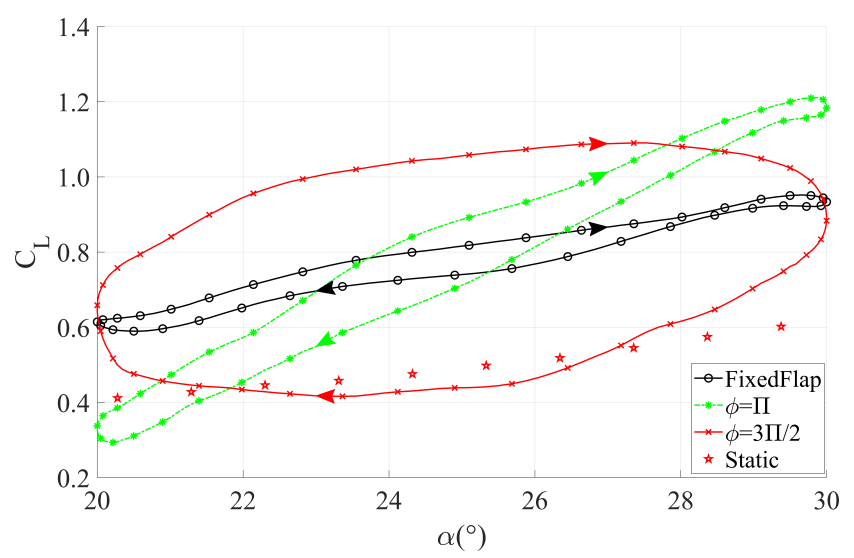

(c) Coefficient of lift $(\phi=1 \pi, \phi=3 \pi / 2)$

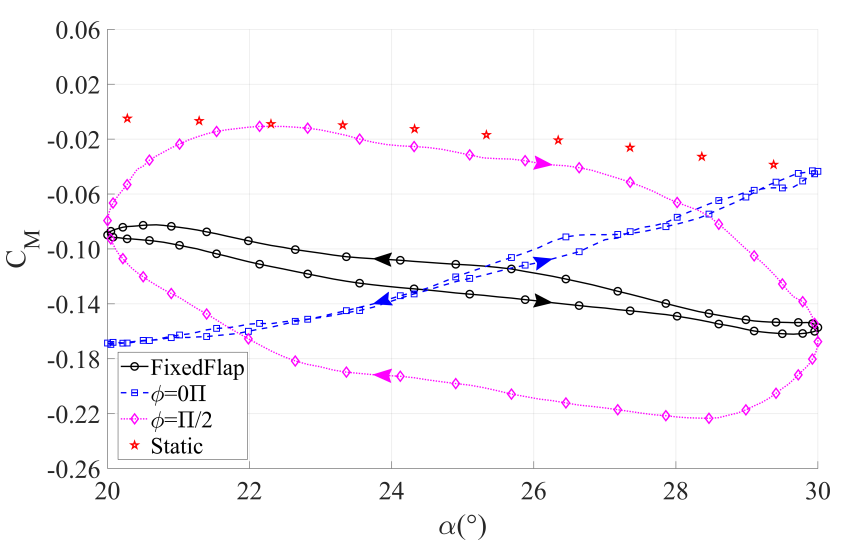

(b) Coefficient of moment $(\phi=0 \pi, \phi=\pi / 2)$

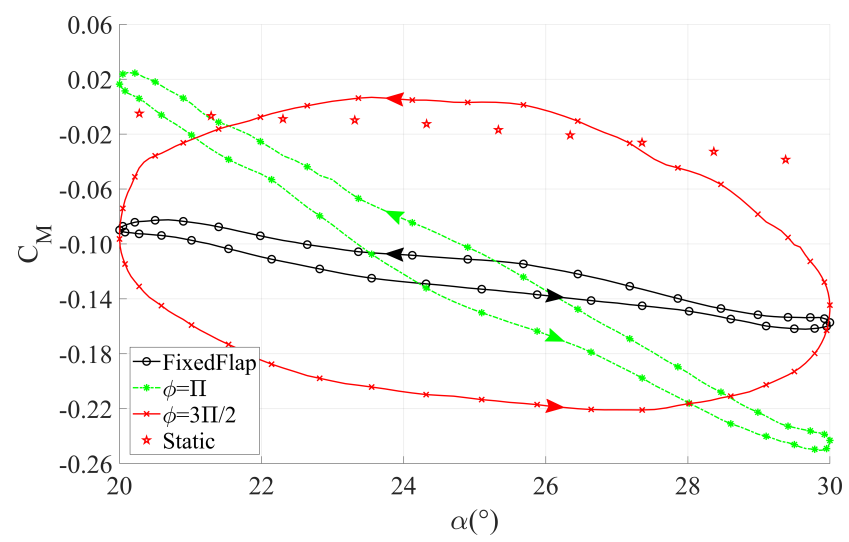

(d) Coefficient of moment $(\phi=1 \pi, \phi=3 \pi / 2)$

Figure 15. Coefficient of lift $\left(C_{L}\right)$, and moment $\left(C_{M}\right)$ versus $\alpha$ for different flap phases $(\phi)$ for case 2 where $k=0.1$, $\alpha_{\text {mean }}=25^{\circ}$, and $\alpha_{a m p}=5^{\circ}$. Only every $4^{\text {th }}$ data point is shown for clarity.

minor subsequent LEV where changes in $\phi$ were responsible for promoting or delaying the occurrence of the LEV in the cycle by $1^{\circ}$. The formation of multiple LEV is in agreement with previous studies. The $C_{M}-\alpha$ loops showed that as the LEV sweeps over the airfoil, it produces a rapid aft movement of the center of pressure from the leading edge towards the trailing edge resulting in a large nose-down pitching moment on the airfoil. The unique contour plots presented in this paper showed the suction peak locations, the primary and secondary LEV formation and the flow separation on the airfoil. It was found that as the LEV is shed, it tends to decrease $-\Delta C_{p}$ close the LE but increases $-\Delta C_{p}$ on the rest of the airfoil surface thus moving the center of pressure.

Two different $k$ values, 0.06 and $\mathbf{0 . 1}$, were tested to represent two different spanwise locations on a yawed wind turbine blade. When $k$ increased from 0.06 to $0.1 \mathrm{a}$ few significant differences arose in the hysteresis loops: the amplitude of the $C_{L}$ peaks caused by LEV are increased and the occurrence of the LEV is delayed by $3^{\circ}$; the difference between the upstroke and downstroke increases, widening the hysteresis loop and increasing load fluctuations; the occurrence of the clockwise loop, that promotes flutter, is delayed by $3^{\circ}$ and the magnitude of negative damping is about the same. Out of all the different $\phi$ tested, it could be concluded that when $\phi=\pi / 2$, $C_{L}$ and $C_{M}$ have the least fluctuations reducing the magnitude of the peaks caused by the LEV and making the hysteresis loops narrower. Normalized power spectral density (PSD) distribution for $C_{L}$ and root bending moment were presented to determine with absolute certainty which $\phi$ case reduces the load fluctuations the most. It was found that for $\phi=\pi / 2, C_{L}$ PSD amplitude is reduced by at least $26 \%$ while the root bending moment PSD amplitude is reduced by at least $24 \%$. It can be concluded that the TEF is not capable of eliminating the LEV completely but it can reduce its impact on the airfoil. 

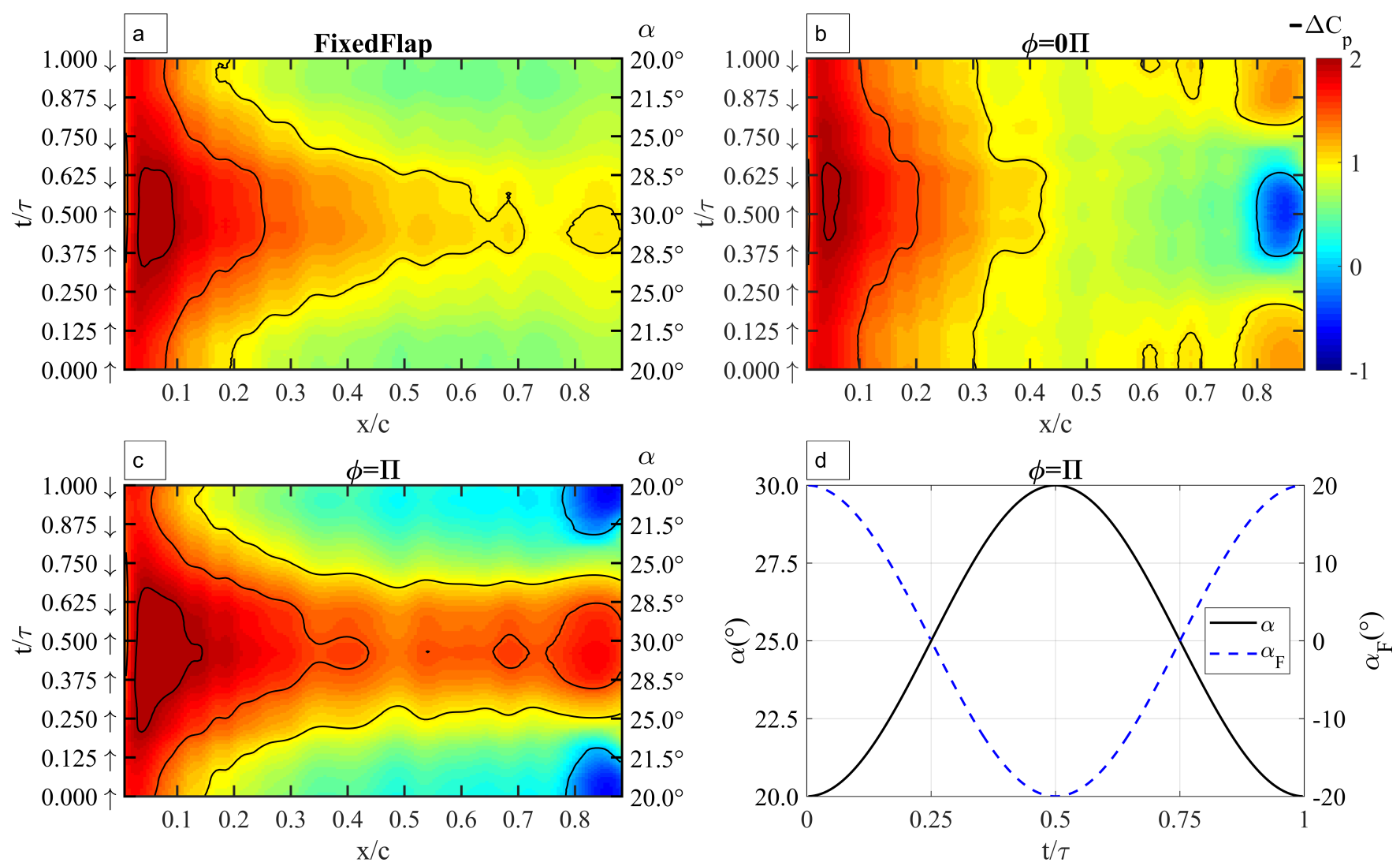

Figure 16. Contour plots showing $-\Delta C_{p}$ versus $\mathrm{x} / \mathrm{c}$ versus $t / \tau$ for case 2 where $k=0.1, \alpha_{\text {mean }}=25^{\circ}$ and $\alpha_{a m p}=5^{\circ}$. a), b), and c) represent FixedFlap, $\phi=0 \pi$, and $\phi=1 \pi$ respectively. d) shows $\alpha$ versus pitch period and flap angle for phase of $1 \pi$. The isobars on the plot represent constant $\Delta C_{p}$ values.

Case 2 in this paper is aimed to simulate a wind turbine blade section operating in yawed condition with a pitching amplitude and mean equivalent to what was found in the literature. What was most interesting about the different loops is that the airfoil is stalled during the entire cycle but even so, $C_{L}$ was higher than its static counterpart. The hysteresis loops for different $\phi$ cases showed that the TEF can manipulate $C_{L}$ at any point on the cycle and that point is determined by $\phi$.

The data presented throughout this paper will contribute towards continued development of TEF and its influence over the dynamic stall cycle. More experimental data and analysis are needed to determine the influence of a TEF over the dynamic stall cycle for a cambered airfoil. The deep dynamic stall cases presented where the pitch angle reaches $30^{\circ}$ are specially unique and novel with important applications to wind turbines and have not been previously reported in the literature. It is anticipated that the discussed experimental data will serve as one of many instruments for gauging, designing, analyzing and optimizing the use of TEF to reduce load fluctuations on wind turbine blades.

\section{Acknowledgements}

We acknowledge the support of the Natural Sciences and Engineering Research Council of Canada (NSERC) through a grant to the second author.

\section{References}

Abbott IH and Doenhoff AEV (1959) Theory of Wing Sections: Including a Summary of Airfoil Data. Dover Publication Inc. Abbott IH and Greenberg H (1939) Tests in the variable-density wind tunnel of the NACA 23012 airfoil with plain and split flaps. National Advisory Committee for Aeronautics NACA-TR-66(NACA-TR-661).

Baik YS, Bernal LP, Granlund K and Ol MV (2012) Unsteady force generation and vortex dynamics of pitching and plunging aerofoils. Journal of Fluid Mechanics 709: 37-68. DOI:https://doi.org/10.1017/jfm.2012.318. 
Barlas TK and van Kuik GAM (2010) Review of state of the art in smart rotor control research for wind turbines. Progress in Aerospace Sciences 46(1): 1-27. DOI:https://doi.org/10.1016/j.paerosci.2009.08.002.

Burton T, Jenkins N, Sharpe D and Bossanyi E (2011) Wind Energy Handbook. Second edition. Wiley.

Carr LW, McAlister KW and McCroskey WJ (1977) Analysis of the Development of Dynamic Stall Based on Oscillating Airfoil Experiments. NASA TN D-8382: 59-64.

Cebeci T, Platzer M, Chen H, Chang KC and Shao JP (2005) Analysis of Low-Speed Unsteady Airfoil Flows. Springer New York. ISBN 3540229329.

Corke TC and Thomas FO (2015) Dynamic Stall in Pitching Airfoils: Aerodynamic Damping and Compressibility Effects. Annual Review of Fluid Mechanics 47(1): 479-505. DOI:https://doi.org/10.1146/annurev-fluid-010814-013632.

Dykes K, Veers P and Lantz E (2019) IEA Wind TCP: Results of IEA Wind TCP Workshop on a Grand Vision for Wind Energy Technology. Technical Report April, National Renewable Energy Laboratory (NREL).

Feszty D, Gillies EA and Vezza M (2004) Alleviation of Airfoil Dynamic Stall Moments via Trailing-Edge Flap Flow Control. AIAA Journal 42(1): 17-25. DOI:https://doi.org/10.2514/1.853.

Gallant T and Johnson DA (2017) Flow angle measurement of a yawed turbine and comparison to models. Wind Energy Science Discussions 2(January): 1-17. DOI:10.5194/wes-2016-57.

Gerontakos P (2004) An Experimental Investigation of Flow Over an Oscillating Airfoil. PhD Thesis, McGill University.

Gharali K and Johnson DA (2013) Dynamic stall simulation of a pitching airfoil under unsteady freestream velocity. Journal of Fluids and Structures 42: 228-244. DOI:https://doi.org/10.1016/j.jfluidstructs.2013.05.005.

Gharali K and Johnson DA (2015) Effects of nonuniform incident velocity on a dynamic wind turbine airfoil. Wind Energy 18(2): 237-251. DOI:https://doi.org/10.1002/we.1694.

Gharali Kb, Johnson D, Lam V and Gu M (2015) A 2D blade element study of a wind turbine rotor under yaw loads. Wind Engineering 39(5): 557-568. DOI:10.1260/0309-524X.39.5.557.

Green R, Gillies E and Wang Y (2011) Trailing edge flap flow control for dynamic stall. Aeronautical Journal 115(1170): 493-503. DOI:https://doi.org/10.1017/S0001924000006138.

Houghton E and Carpenter P (2002) Aerodynamics for Engineering Students. Fifth edition. Butterworth Heinemann.

Jacobs EN and Pinkerton RM (1931) Pressure Distribution Over a Symmetrical Airfoil Section with Trailing Edge Flap. National Advisory Committee for Aeronautics (Report No. 360).

Lee T and Gerontakos P (2004) Investigation of flow over an oscillating airfoil. Journal of Fluid Mechanics 512: 313-341. DOI:https://doi.org/10.1017/S0022112004009851.

Lee T and Gerontakos P (2006) Dynamic Stall Flow Control via a Trailing-Edge Flap. AIAA Journal 44(3): 469-480. DOI: https://doi.org/10.2514/1.17263.

Lee T and Su YY (2011) Unsteady airfoil with a harmonically deflected trailing-edge flap. Journal of Fluids and Structures 27(8): 1411-1424. DOI:https://doi.org/10.1016/j.jfluidstructs.2011.06.008.

Leishman JG (1990) Dynamic stall experiments on the NACA 23012 aerofoil. Experiments in Fluids 9(1-2): 49-58. DOI: https://doi.org/10.1007/BF00575335.

Leishman JG (2006) Principles of Helicopter Aerodynamics. Second edition. Cambridge University Press.

Masdari M, Seyednia M and Tabrizian A (2018) An experimental loading study of a pitching wind turbine airfoil in nearand post-stall regions. Journal of Mechanical Science and Technology 32(8): 3699-3706. DOI:https://doi.org/10.1007/ s12206-018-0722-7.

McCroskey WJ (1981) The Phenomenon of Dynamic Stall. DTIC Document NASA .

McCroskey WJ (1982) Unsteady Airfoils. Annual Review of Fluid Mechanics 14:285-311: 285-311. DOI:https://doi.org/10. 1146/annurev.fl.14.010182.001441.

McCroskey WJ, Carr LW and McAlister KW (1976) Dynamic Stall Experiments on Oscillating Airfoils. AIAA Journal 14(1): 57-63. DOI:https://doi.org/10.2514/3.61332.

Morote J (2016) Angle of attack distribution on wind turbines in yawed flow. Wind Energy 19(4): 681-702. DOI: https://doi.org/10.1002/we.1859.

Pitt Ford CW and Babinsky H (2013) Lift and the leading-edge vortex. Journal of Fluid Mechanics 720: 280-313. DOI: https://doi.org/10.1017/jfm.2013.28.

Raiola M, Discetti S, Ianiro A, Samara F, Avallone F and Ragni D (2018) Smart Rotors: Dynamic-Stall Load Control by Means of an Actuated Flap. AIAA Journal 56(4): 1-14. DOI:https://doi.org/10.2514/1.J056342.

Samara F (2020) Active Aerodynamic Modification of Wind Turbine Blades to Reduce Load Fluctuation. Phd thesis, University of Waterloo. URL http://hdl.handle.net/10012/15760. 
Samara F and Johnson DA (2018) In-blade Load Sensing on 3D Printed Wind Turbine Blades Using Trailing Edge Flaps. Journal of Physics 1037: 052023. DOI:10.1088/1742-6596/1037/5/052023.

Samara F and Johnson DA (2020a) Dynamic Stall on Pitching Cambered Airfoil with Phase Offset Trailing Edge Flap. AIAA Journal : 1-13DOI:10.2514/1.J059115.

Samara F and Johnson DA (2020b) In-Blade Measurements of Cyclic Loading on Yawed Turbines with Trailing Edge Flap. Journal of Physics 1452(1): 012061. DOI:10.1088/1742-6596/1452/1/012061.

Shehata I, Zakaria MY, Hussein A and Hajj MR (2018) Aerodynamic analysis of flapped airfoil at high angles of attack. AIAA Aerospace Sciences Meeting, 2018 (210059). DOI:10.2514/6.2018-0037.

Shipley DE, Miller MS, Robinson MC, Luttges MW and Simms DA (1995) Dynamic stall occurrence on a horizontal axis wind turbine blade. Technical Report July, National Renewable Energy Laboratory (NREL), Golden, CO (United States). DOI:10.2172/61151. URL http://www.osti.gov/servlets/purl/61151/.

Somers DM (2005) The S833, S834, and S835 Airfoils. National Renewable Energy Laboratory, NREL NREL/SR-50. DOI: $10.2172 / 15020040$.

Troldborg N (2005) Computational Study of the Ris $\varnothing-B 1-18$ Airfoil with a Hinged Flap Providing Variable Trailing Edge Geometry. Wind Engineering 29(2): 89-113. DOI:10.1260/0309524054797159. URL http://journals.sagepub.com/doi/ $10.1260 / 0309524054797159$.

Zanotti A (2012) Retreating Blade Dynamics Stall. Phd thesis, Politecnico di Milano.

Zanotti A and Gibertini G (2013) Experimental investigation of the dynamic stall phenomenon on a NACA 23012 oscillating airfoil. Proceedings of the Institution of Mechanical Engineers, Part G: Journal of Aerospace Engineering 227(9): 13751388. DOI:10.1177/0954410012454100.

Zanotti A and Gibertini G (2018) Experimental assessment of an active L-shaped tab for dynamic stall control. Journal of Fluids and Structures 77: 151-169. DOI:10.1016/j.jfluidstructs.2017.11.010. 